OPEN ACCESS

Edited by:

Yasuhito Shimada,

Mie University, Japan

Reviewed by:

Lisa Crawford

Queen's University Belfast

United Kingdom

Alison L. Reynolds,

University College Dublin, Ireland

*Correspondence:

Jakub K. Famulski

jkfa226@uky.edu

tThese authors have contributed equally to this work

Specialty section:

This article was submitted to

Molecular Medicine

a section of the journal

Frontiers in Cell and Developmental

Biology

Received: 14 August 2020 Accepted: 22 October 2020

Published: 23 November 2020

Citation:

Piedade WP, Titialii-Torres $K$, Morris AC and Famulski JK (2020) Proteasome-Mediated Regulation of Cdhr1a by Siah1 Modulates

Photoreceptor Development and Survival in Zebrafish. Front. Cell Dev. Biol. 8:594290. doi: $10.3389 /$ fcell.2020.594290

\section{Proteasome-Mediated Regulation of Cdhr1a by Siah1 Modulates Photoreceptor Development and Survival in Zebrafish}

\author{
Warlen Pereira Piedade ${ }^{\dagger}$, Kayla Titialii-Torres ${ }^{\dagger}$, Ann C. Morris and Jakub K. Famulski* \\ Department of Biology, University of Kentucky, Lexington, KY, United States
}

Congenital retinal dystrophies are a major cause of unpreventable and incurable blindness worldwide. Mutations in CDHR1, a retina specific cadherin, are associated with cone-rod dystrophy. The ubiquitin proteasome system (UPS) is responsible for mediating orderly and precise targeting of protein degradation to maintain biological homeostasis and coordinate proper development, including retinal development. Recently, our lab uncovered that the seven in absentia (Siah) family of E3 ubiquitin ligases play a role in optic fissure fusion and identified Cdhr1a as a potential target of Siah. Using two-color whole mount in situ hybridization and immunohistochemistry, we detected siah1 and cdhr1a co-expression as well as protein localization in the retinal outer nuclear layer $(\mathrm{ONL})$, and more precisely in the connecting cilium of rods and cones between 3-5 days post fertilization (dpf). We confirmed that Siah1 targets Cdhr1a for proteasomal degradation by co-transfection and co-immunoprecipitation in cell culture. To analyze the functional importance of this interaction, we created two transgenic zebrafish lines that express siah1 or an inactive siah1 (siah1 $\Delta \mathrm{RING)}$ under the control of the heat shock promoter to modulate Siah activity during photoreceptor development. Overexpression of siah1, but not siah1 $\Delta \mathrm{RING}$, resulted in a decrease in the number of rods and cones at $72 \mathrm{~h}$ post fertilization (hpf). The number of retinal ganglion cells, amacrine and bipolar cells was not affected by Siah1 overexpression, and there was no significant reduction of proliferating cells in the Siah1 overexpressing retina. We did, however, detect increased cell death, confirmed by an increase in the number of TUNEL + cells in the ONL, which was proteasome-dependent, as proteasome inhibition rescued the cell death phenotype. Furthermore, reduction in rods and cones resulting from increased Siah1 expression was rescued by injection of colhr1a mRNA, and to an even greater extent by injection of a Siah1-insensitive colhr1a variant mRNA. Lastly, CRISPR induced loss of Cdhr1a function phenocopied Siah1 overexpression resulting in a significant reduction of rods and cones. Taken together, our work provides the first evidence that Colhr1a plays a role during early photoreceptor development and that Cdhr1a is regulated by Siah1 via the UPS.

Keywords: retina, cell death, UPS, zebrafish, photoreceptor, Siah E3 ubiquitin ligase, CDHR1a 


\section{INTRODUCTION}

According to the World Health Organization (WHO), in 2015 more than 253 million people worldwide were visually impaired, of which 36 million were blind. This number is predicted to increase to 703 million visually impaired people by 2050 (Ackland et al., 2017). Retinal congenital disease is a major contributor to blindness disorders, affecting 4.5 million people worldwide. Congenital retinal blindness is known to be associated with mutations in over 280 different genes (Hohman, 2016; Riera et al., 2017; Benati et al., 2019). While these mutations encompass various portions of the eye, aberrant development and improper maintenance of the retina are major causes of visual impairment and loss of sight.

The retina, an extension of the central nervous system, is responsible for not only detecting incoming light, via photoreceptor cells, but also ultimately conveying that signal through the optic nerve and to the brain to be interpreted as vision (Purves et al., 2001). Retinal structure and development are fairly well conserved across vertebrates from human to mouse and zebrafish (Hoon et al., 2014). There are 7 cell types within the retina, which populate 3 nuclear layers. Differentiation of the neural retina generally begins with the innermost neurons of the retina: ganglion cells within the ganglion cell layer (GCL) closely followed by or in parallel with amacrine, horizontal, and bipolar cells of the inner nuclear layer (INL). Müller glia are the last cells of the INL to differentiate (Centanin and Wittbrodt, 2014). The light-sensing rod and cone photoreceptor cells, which are responsible for distinguishing light and dark as well as color, respectively, are relatively lateborn retinal cell types (Kawamura and Tachibanaki, 2008). In zebrafish beginning at $50 \mathrm{~h}$ post fertilization (hpf), expression of rhodopsin and red cone opsin are detectable specifically in the ventronasal region of the retina (Larison and Bremiller, 1990; Schmitt and Dowling, 1996; Fadool, 2003). Cone photoreceptor differentiation then spreads from the ventro-nasal to the dorso-temporal retina in a wave, whereas rod photoreceptor differentiation proceeds more sporadically across the retina (Raymond et al., 1995; Morris and Fadool, 2005). Photoreceptor outer segments are initially detected at $60 \mathrm{hpf}$ in the ventral region of the retina (Avanesov and Malicki, 2010). Cone and rod photoreceptors have largely completed differentiation by 5 days post fertilization (dpf), at which point cone photoreceptor function can be detected by electrophysiology and behavioral assays (Brockerhoff et al., 1995; Fadool and Dowling, 2008). Photoreceptors populate the outer nuclear layer (ONL) and play a critical role in detecting light using the outermost portion of the cell, the outer segment (Baker and Kerov, 2013). Outer segments are comprised of hundreds of stacked disks which contain the molecular machinery to detect and process light signals via phototransduction (Goldberg et al., 2016). Phototransduction is a highly metabolically demanding process which produces toxic photo-oxidative compounds, requiring outer segments to shed after a period of time and be replenished to maintain proper cell length (Kocaoglu et al., 2016). Although photoreceptors are imperative for visual system operation, the function of many genes hypothesized to play a role in their development and maintenance have yet to be tested in vivo. Mutations in these various genes can lead to the development of a wide spectrum visual impairment, including the commonly known cone-rod dystrophies.

Cone-rod dystrophies are a group of inherited retinal diseases that first affect cone photoreceptors, then rod photoreceptors, or in some cases they are affected simultaneously (Hamel, 2007). Generally, the photoreceptors begin to degenerate, causing progressive loss in visual acuity, color and central vision, and light sensitivity (Gill et al., 2019). In order to develop therapeutics for cone-rod dystrophies, understanding the currently unknown mechanism as to how of each of the over 30 genes (Sullivan et al., 2020) implicated in its onset and progression is imperative. To do this, most studies have aimed to elucidate the role of these genes in retinal development and maintenance in vertebrate models such as mouse and zebrafish (Biehlmaier et al., 2003; Parry et al., 2009; Buch et al., 2011; Lu et al., 2017; Qu et al., 2019). A well-established candidate gene associated with conerod dystrophy which has yet to be explored in a developmental context is photoreceptor specific cadherin CDHR1.

Several clinical studies (Bolz et al., 2005; Henderson et al., 2010; Ostergaard et al., 2010; Cohen et al., 2012; Duncan et al., 2012; Ba-Abbad et al., 2013; Bessenyei and Oláh, 2014; Nikopoulos et al., 2015; Arno et al., 2016; Riera et al., 2017; Stingl et al., 2017; Fu et al., 2018) have described mutations in CDHR1 associated with inherited cone-rod dystrophy. Conserved among vertebrates, CDHR1 belongs to the cadherin superfamily of calcium-dependent cell adhesion molecules but is exclusively expressed in photoreceptors of zebrafish, chickens, mice, and humans (Rattner et al., 2004). CDHR1 encodes an intracellular domain, a transmembrane domain in addition to six cadherin repeats (Stingl et al., 2017). Previous studies using tomography, electron microscopy and immunohistochemistry (IHC) have unequivocally defined CDHR1 localization to the base of outer segment of photoreceptor cells (Rattner et al., 2001, 2004; Burgoyne et al., 2015). Additionally, to further determine its precise location in the junction in between the inner segment (IS) and the outer segment (OS), Burgoyne et al. (2015) used nanogold cryo-EM to illustrate that CDHR1 forms fibers connecting immature disks at the base of the outer segment. This group hypothesized that CDHR1 is necessary to stabilize and control the disk evagination process during photoreceptor cell outer segment assembly and/or maintenance (Ostergaard et al., 2010). A CDHR1 knockout mouse partially supports this hypothesis as well as the correlation of CDHR1 loss of function and conerod dystrophy. CDHR1 knockout mice were born with shorter and disorganized photoreceptor outer segments, followed by a progressive loss of photoreceptors (50\%) in the next 6 months of life (Rattner et al., 2001).

While previous studies of CDHR1 have confirmed its importance for photoreceptor development and homeostasis (Nikopoulos et al., 2015) we lack any understanding of its regulation during these critical events. Interestingly, we have recently characterized a ubiquitin-proteasomal system (UPS) pathway involved in retinal morphogenesis (Piedade et al., 2019). We observed that the E3 ligase enzyme, Siah1, was expressed throughout the retina during early development 
and specifically targeted a transcriptional regulator, Nlz2, for degradation. This process ensured timely and precise fusion of the optic fissure of the early retina. When searching for other targets of this E3 ligase based on its well established degron-motif (Pro-[ARTE]-X-Val-X-Pro), we identified zebrafish Cdhrla as a potential hit. This suggested to us that Siah is a candidate for regulating the turnover of this protein and therefore controlling its function during photoreceptor development or outer segment maintenance.

In our present study we aimed to investigate the Siahmediated post-translational regulation of Cdhrla during development of the zebrafish retina. Taken together, our data indicate that stability of Cdhrla is necessary for zebrafish photoreceptor development and survival and it is subject to regulation through the UPS by Siah1. In particular, we observe significantly reduced photoreceptor number upon induced expression of Siah, but no significant effects on any other retinal cell type. We show these effects are UPS dependent and can be rescued with a proteasome inhibitor (MG132), with cdhrla mRNA, as well as a Siah1 insensitive Cdhrla variant. Our work provides an in vivo example of vertebrate photoreceptor cell development modulated by UPS-mediated regulation of a gene known to be associated with inherited cone-rod dystrophy.

\section{RESULTS}

\section{Siah and cdhr1a Are Co-localized in the Outer Nuclear Layer During Retinal Development}

As mentioned above, numerous studies have demonstrated $c d h r 1$ expression in retinal photoreceptor cells and potentially implicated in photoreceptor development (Rattner et al., 2001, 2004; Ostergaard et al., 2010). In contrast, siah gene expression during zebrafish retinal development had yet to be described. As such, we sought to investigate siah expression and cellular localization during the later stages of retinal development when photoreceptors are maturing. Siah1 and Siah2l are known to be expressed throughout the central nervous system during zebrafish development but in this study we carried out a comprehensive expression analysis of both siah homologues, siah1 and siah2l specifically during retinal development. Using two color fluorescence whole-mount in situ hybridization (FWISH) we examined simultaneous expression of siah1 or siah $2 l$ and cdhrla in the zebrafish retina at 3, 4, and 5 days post fertilization (dpf; Figure 1). Zebrafish also encode a cdhr1 paralogue, cdhr1b, however, it does not exhibit retinal expression (data not shown). Starting at $3 \mathrm{dpf}$ we observed co-expression of cdhr1a and both siah1 and siah2l specifically in the ONL (Figures 1A,D). Siah1 and Siah2l expression were also seen throughout the INL and GCL. This pattern of expression was observed up to and including $5 \mathrm{dpf}$ (Figures 1C,F). Coexpression of siah1 and cdhrla in the ONL indicates that siahl and cdhrla are both present and potentially active during photoreceptor cell maturation. This further suggests that Siah1 may have a functional role in regulating Cdhrla protein stability in photoreceptor cells. Since siah1 and siah $2 l$ both target the same degron sequence, we focused solely on siah1 for the rest of this study.

\section{Siah1 Localizes to the Base of the Outer Segments in Rods and Cones}

In order to validate our FWISH results, we next examined Siah and Cdhrla protein localization during photoreceptor maturation. To do so we first obtained zebrafish specific polyclonal antibodies against Siah1 and Cdhrla. When tested by IHC in 3-5 dpf retinal sections we observed signal that correlated with our FWISH results (Figures 2A-L). In particular, we observed specific localization of Cdhrla signal in the ONL where the rods and cones reside, while Siah1 signal was detected throughout the retina, including strong signal in the ONL (Figures 2D-F). To confirm that Siah1 is localized in photoreceptor cells, and to determine to which subcellular region, we performed IHC on retinal sections from transgenic embryos expressing rod and cone reporter constructs, $\operatorname{Tg}[X O P S: G F P]$ and Tg[T $\alpha$ C:eGFP], respectively, (Fadool, 2003; Kennedy et al., 2007). Our IHC results indicate that Siah1 protein localized to the synaptic terminals of rod (XOPS:GFP) and cone ( $T \alpha C$ :eGFP) photoreceptors, as well as in the connecting cilium from 3 to 5 dpf (Figures 2D'-F',J'-K',C”,F”,I",L”). We observed a similar pattern of localization for Cdhrla, in particular at the connecting cilium of rods (XOPS:GFP) and cones (TaC:eGFP; Figures $2 \mathbf{A}^{\mathbf{\prime}}-$ C',G'-H'). Cdhrla localization to the primary cilium at $3 \mathrm{dpf}$, exhibited low signal, which increased progressively up to $5 \mathrm{dpf}$ (Figures 2C",F",I",L"). In addition to the ONL, Siah1 protein staining was also observed in the INL and GCL during the period analyzed. This again corelated with our FWISH data. Collectively, our analysis of mRNA and protein localization for Siah and Cdhrla indicated that both proteins are expressed in photoreceptor cells and may functionally interact.

\section{Siah1 Targets Cdhr1a for Proteasomal Mediated Degradation}

Siah E3 ubiquitin ligase interaction with target proteins is a well-characterized process and it involves interaction through an evolutionarily conserved amino acid motif termed a degron. Zebrafish Cdhrla protein encodes a Siah degron starting in the 857th amino acid. In vertebrates, CDHR1 is highly conserved, with similarity ranging around $60 \%$ when comparing human to zebrafish Cdhrla. This includes the degron motif, suggesting that this conserved feature plays an important role in the regulation of Cdhrla through the UPS (Figure 3A). To examine whether Siah regulates Cdhrla protein degradation, we transiently co-transfected HEK 293T cells with CdhrlaFLAG and GFP (control) or Cdhrla-FLAG and Siah1-myc. Protein levels were subsequently determined by Western blot. As shown in Figure 3B, lysates from cells co-transfected with Cdhrla-FLAG and Siah1-myc, completely lacked Cdhrla-FLAG signal while the control co-transfection resulted in the presence of a strong Cdhrla-FLAG band (Figure 3B), indicating that Cdhrla is targeted for degradation in the presence of Siah1. To demonstrate a direct effect of Siah1 E3 ligase activity 


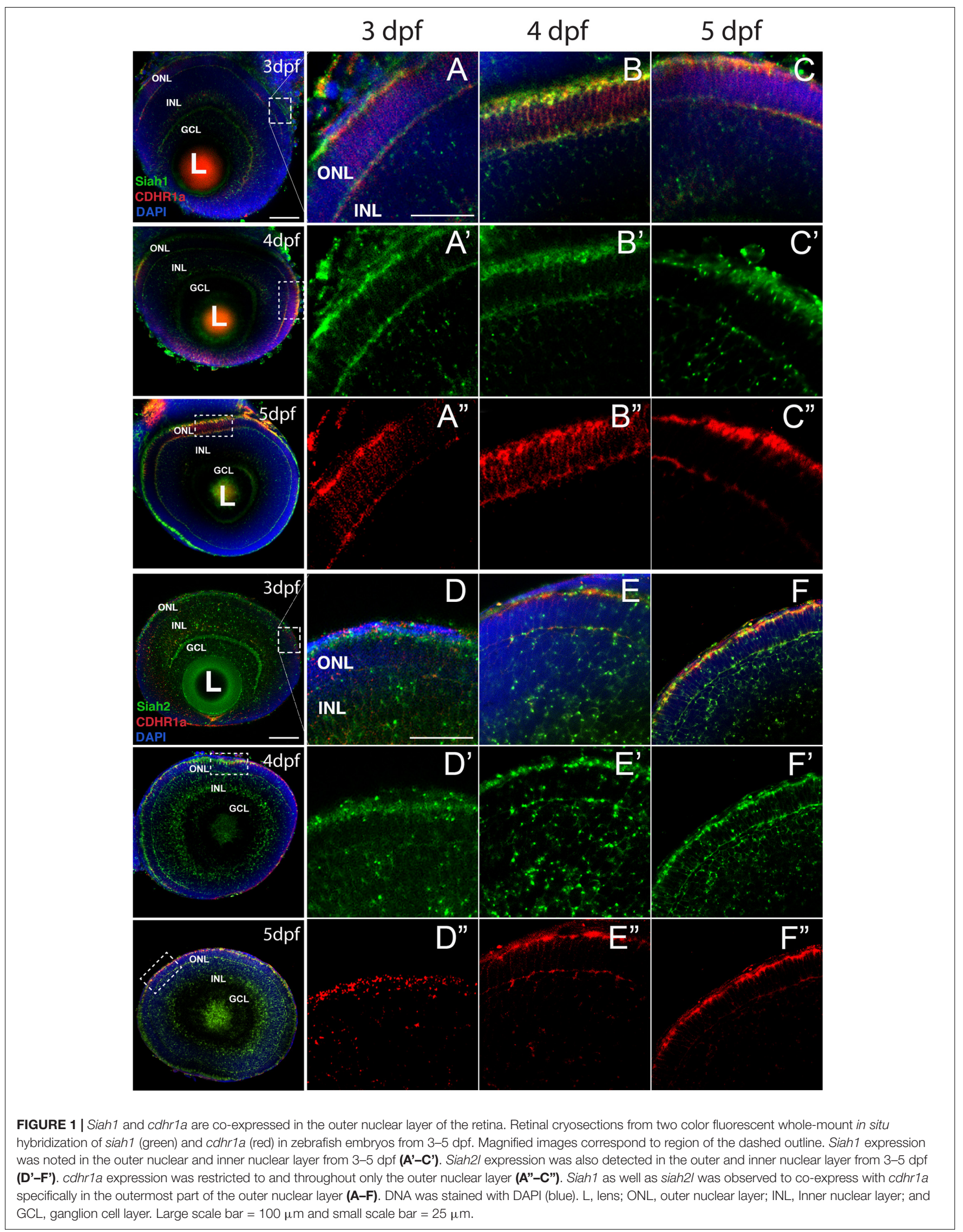



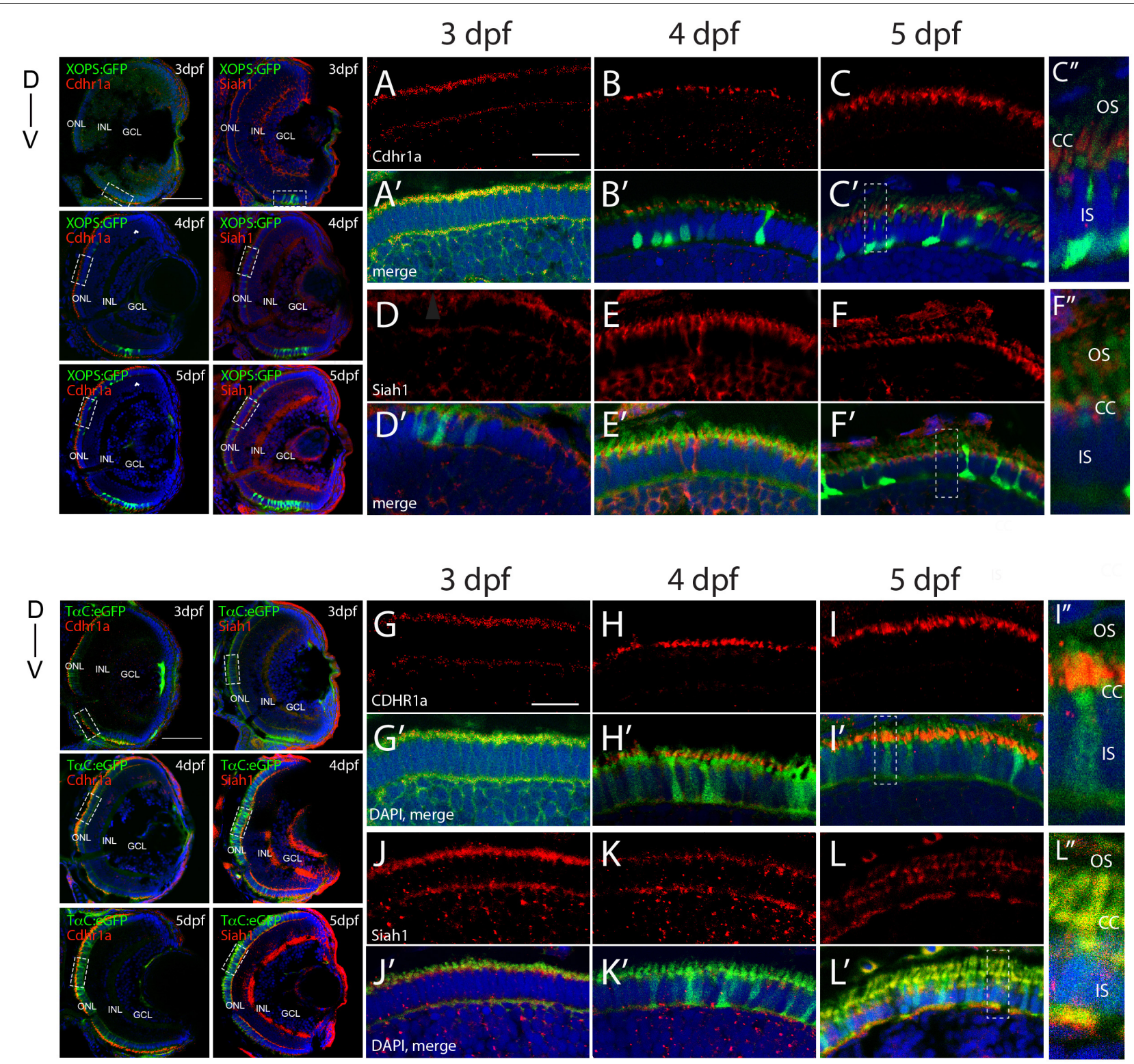

FIGURE 2 | Siah1 and Cdhr1a localize to the photoreceptor primary cilium. Siah1 and Cdhr1a protein localization was determined using IHC in 3-5 dpf old Tg[XOPS:GFP] or Tg[TaC:eGFP] retinal cryosections. Cdhr1a signal (red) was detected in the ONL (A-C, G-I) and within rod photoreceptors (green) marked by XOPS:GFP expression between 3-5 dpf (A'-C'). Siah1 signal was also detected in the ONL (D,E,J-L) and within rod photoreceptors ((D', E'). Cdhr1a signal (red) was detected within cone photoreceptors (green) marked by TaC:eGFP expression between 3-5 dpf (J'-L'). Siah1 signal was also detected within cone photoreceptors ('D',E'). Both Cdhr1a and Siah1 localization within photoreceptors was strongest at the junction of the inner and outer segments, connecting cilium (C",F",I",L"). DNA was stained with DAPI (blue). L, lens; ONL, outer nuclear layer; INL, Inner nuclear layer; GCL, ganglion cell layer; D, Dorsal; and V, Ventral; OS, outer segment; CC, connecting cilium; and IS, inner segment. Large scale bar $=100 \mu \mathrm{m}$ and small scale bar $=10 \mu \mathrm{m}$.

we also co-transfected Cdhrla-FLAG with an inactivated Siah1 construct (Siah1 $\Delta$ RING-myc) which is missing the RING domain and therefore cannot perform the E3-mediated ubiquitin transfer onto its targets. Cell lysate from CdhrlaFLAG and Siah1 $\triangle$ RING-myc co-transfection also contained a strong Cdhrla-FLAG band. Furthermore, inhibition of the proteasome using MG132 treatment resulted in the retention Cdhrla-FLAG signal compared to no treatment (Figure 3B). These results demonstrated that Siah1 is directly responsible for the loss of Cdhrla-FLAG due to proteasomal degradation. To determine whether Cdhrla targeting by Siah1 requires the degron motif, we constructed a Cdhrla variant in which the $\mathrm{VmP}$ motif of the degron sequence was altered to LmA $\left(c d h r 1 a^{\mathrm{LmA}}\right)$. The mutations had no effect on the level of expression of Cdhrla ${ }^{\text {LMA }}$-FLAG compared to Cdhrla-FLAG (Figures 3B,C). As shown in Figure 3C, Cdhrla ${ }^{\mathrm{LmA}}-\mathrm{FLAG}$ was completely insensitive to the effects of co-expressing Siah1-myc. Finally, using co-immunoprecipitation (co-IP) we 


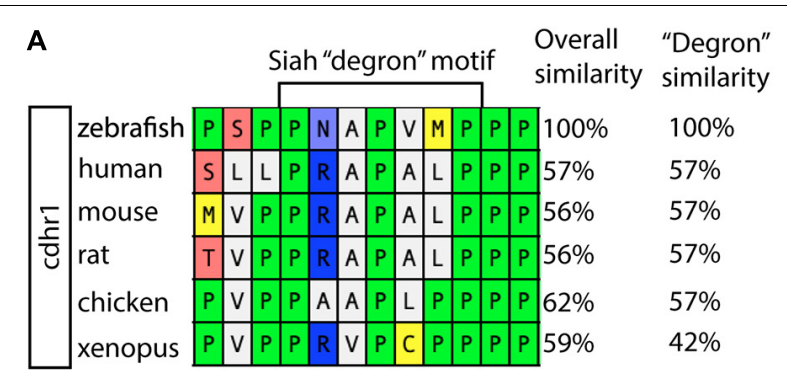

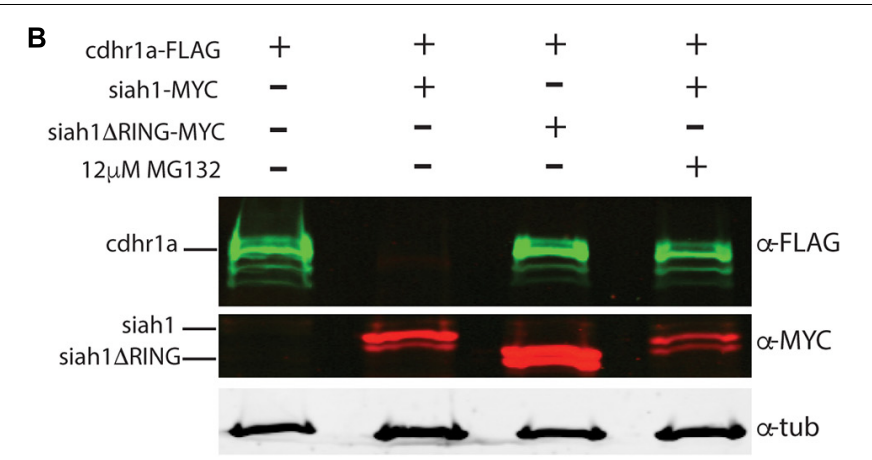

D

C

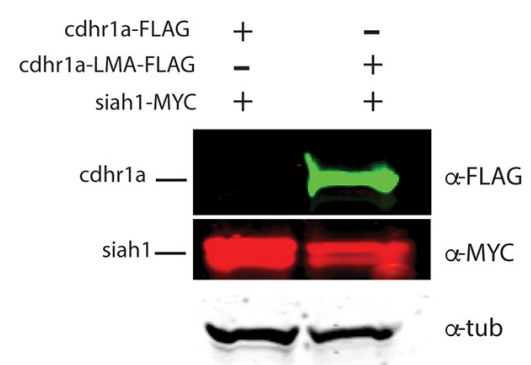

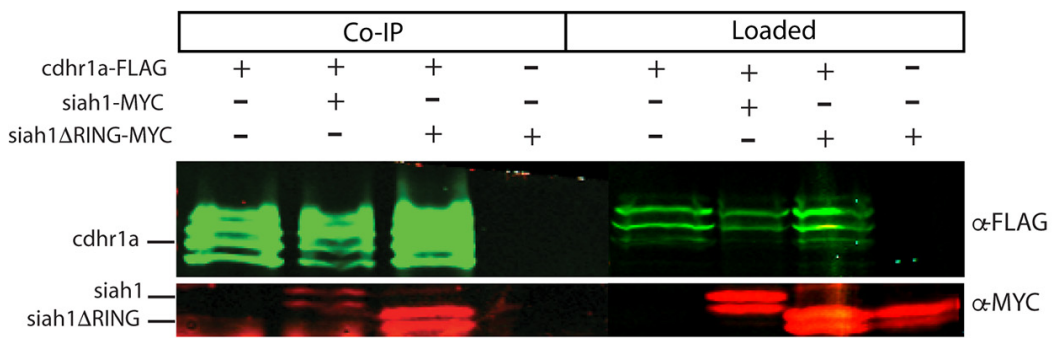

FIGURE 3 | Siah1 targets Cdhr1a for proteasomal degradation. Alignment of CDHR1 degron motif sequence from different vertebrates: Xenopus, chicken, mouse, rat, human, and zebrafish outlining overall protein sequence as well as motif conservation (A). Western blot analysis of cdhr1a protein stability in response to Siah activity. cohr1a-FLAG signal is significantly decreased by co-transfection of siah1-myc, but not siah1 $\Delta$ R-myc, or upon MG132 treatment. Alpha/betta tubulin was used as a loading control. $N=3$ independent transfection experiments (B). Western blot analysis of Siah1 targeting specificity. colhr1a-FLAG signal is significantly decreased by co-transfection of siah1-myc. Signal of cdhr1a ${ }^{\mathrm{LMA}}$-FLAG, a cdhr1a variant encoding a non-recognized degron motif, does not decrease upon co-transfection of siah1-myc. Alpha tubulin was used as a loading control. $N=3$ independent experiments (C). Co-immunoprecipitation of cdhr1a-FLAG co-transfected with siah1-myc or siah1 $\Delta$ R-myc probed for FLAG (green), MYC (red). Cdhr1a-FLAG is able to pull down both siah1 and siah1 $\Delta$ RING. $N=2$ independent experiments (D).

showed that Siah1-myc, or Siah1 $\Delta$ R-myc, can be pulled down by Cdhrla-FLAG (Figure 3D). To ensure the specificity of our co-IP, cells were transfected with Siah1 $\Delta$ RING-myc alone, showing no pull-down with FLAG antibodies after the coIP (Figure 3D). Taken together, these results strongly suggest that Siah1 directly targets Cdhrla for proteasomal mediated degradation through the degron motif found in Cdhrla. In light of our findings, we next sought to determine whether Siahmediated regulation of Cdhrla protein stability plays a role in zebrafish photoreceptor development.

\section{Misregulation of Siah1 Activity Leads to Reduced Numbers of Photoreceptors}

Based on our characterization of siah 1 and $c d h r 1 a$ expression and localization in photoreceptor cells, specifically the connecting cilium, and our confirmation that Siah1 targets Cdhrla for degradation in vitro, we next wanted to determine whether this interaction plays a functional role during photoreceptor cell development. In order to overexpress Siah1 during retinal development, we generated two zebrafish transgenic lines in which Siah1 or the inactive Siah1 $\triangle$ RING were placed under the control of the heat shock (HS) inducible $h s p 70$ promoter (Figure 4A). We designed an experimental HS approach that would induce Siah1 expression during the developmental window of photoreceptor genesis, between 48 and $72 \mathrm{~h}$ post fertilization (hpf). To ensure continuous activity of the transgene, we performed the initial HS at $48 \mathrm{hpf}$, followed by a repeat $\mathrm{HS}$ at $60 \mathrm{hpf}$, and finally fixation at $72 \mathrm{hpf}$, by which time photoreceptor differentiation is largely completed (Figure 4B). The efficiency and specificity of the HS system were assessed by whole-mount in situ hybridization (WISH; Figure 4C). In the absence of elevated temperature examination of siah1 expression did not suggest any leaky expression from the $h s p 70$ promoter. Upon HS siah1 and siah1 $\triangle$ RING expression were significantly and ubiquitously elevated (Figure 4C). Importantly, HS mediated induction of siah1 but not siah1 $\triangle$ RING expression led to a decrease of cdhrla levels in the ONL as observed using IHC (Figure 4D). $\operatorname{Tg}[h s p 70: s i a h 1]$ and $\operatorname{Tg}[h s p 70: \operatorname{siah} 1 \Delta \mathrm{RING}]$ were next crossed onto the $\operatorname{Tg}[X O P S: G F P]$ and $\operatorname{Tg}[T \alpha C: \mathrm{eGPP}]$ transgenic lines to assess rod and cone photoreceptor development, respectively. At this timepoint in development, rod photoreceptors are largely concentrated in the ventral portion of the retina with some sporadic differentiation in the dorsal retina. In contrast, cone photoreceptors, which complete differentiation faster than the rods, have spread fairly uniformly across the ONL by 72 hpf. Double transgenic embryos were subjected to the HS protocol. Collected embryos were either imaged whole using confocal microscopy, or cryosectioned for IHC analysis. Confocal imaging of whole-mount embryos clearly 
A

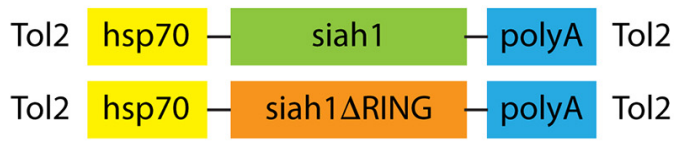

C

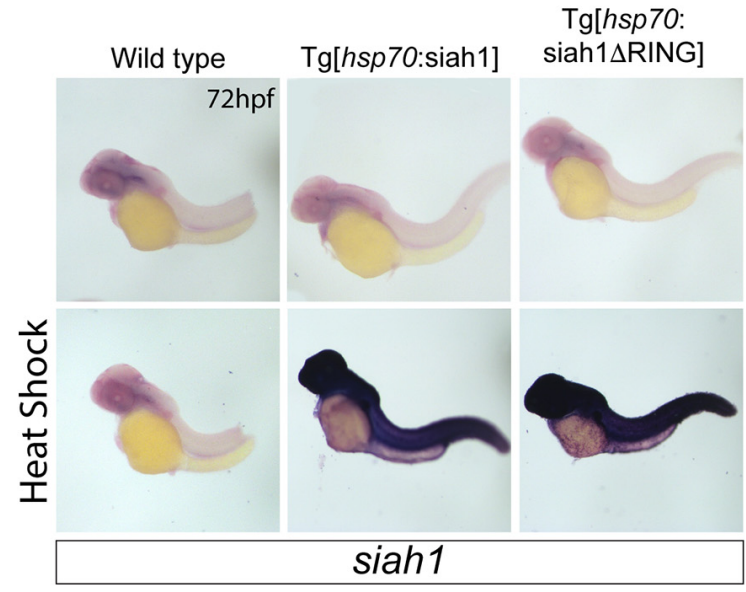

B

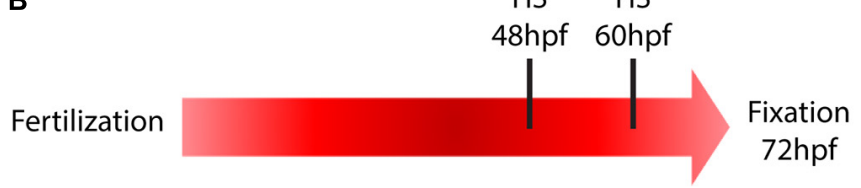

D

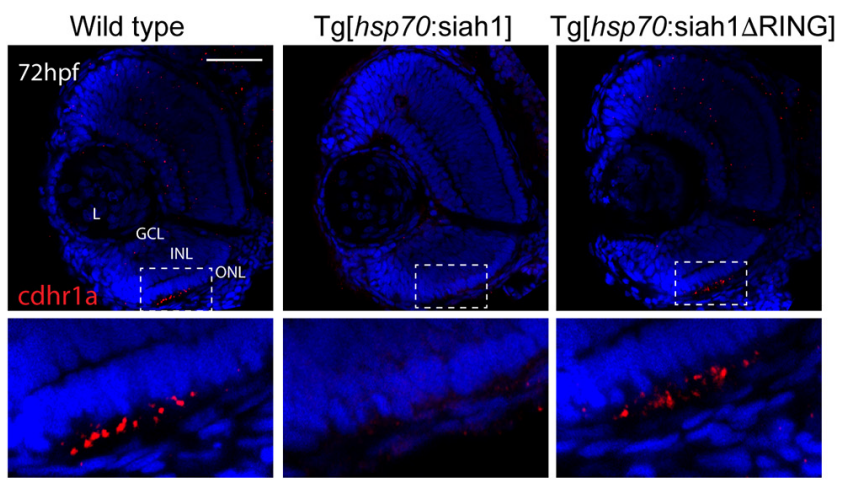

FIGURE 4 | Siah overexpression experimental design. Heat shock line construct schematic (A). The experimental design included heat shock for 30 min at 48 and subsequently $60 \mathrm{hpf}$ with fixation and analysis at $72 \mathrm{hpf} \mathbf{( B )}$. Whole-mount in situ hybridization (WISH) for siah1 to confirm the effect of heat shock in wildtype, Tg[hsp70:Siah1] and Tg[hsp70:Siah1 $\Delta$ RING] embryos. Heat shock induced a significant increase in siah1 gene expression in the transgenic lines but not in wildtype (C). Cdhr1a protein localization (red) was determined using IHC in retinal cryosections from 3 dpf old heat shocked wildtype, Tg[hsp70:Siah1] or Tg[hsp70:Siah1 $\triangle$ RING] embryos. DNA was stained with DAPI (blue). Scale bar = 50 $\mu \mathrm{m}$. L, lens; ONL, outer nuclear layer; INL, Inner nuclear layer; and GCL, ganglion cell layer.

indicated a significant decrease in rod and cone cells in Siah1 overexpressing embryos at $72 \mathrm{hpf}$ relative to controls (Supplementary Figure 1). Quantification of retinal sections confirmed a decrease in the number of rods (Figures $5 \mathbf{A}-\mathbf{C}$ ) and cones (Figures 5E-G) in Siah1 HS embryos compared to wildtype and siah1 $\triangle$ RING HS (Figure 5). The decrease in mature rod photoreceptors in the $\mathrm{Tg}[X O P S$ GFP] line was most evident in the ventral portion of the retina (Figures $5 \mathbf{A}-\mathbf{C}^{\prime}$ ), where rod photoreceptors initially differentiate (Morris et al., 2008; Figure 5D). Rods in the wildtype HS embryos displayed an elongated cell shape with partially visible outer segments (Figure 5A') whereas rods in the Siah1 HS embryos appeared wider and without visible outer segments (Figure 5'C'). Rods in Siah1 $\triangle$ RING HS embryos retained wildtype numbers and morphology (Figures 5B',D). Some rods appeared stunted in shape, but most had an elongated structure with visible outer segments, comparable to wildtype HS. When examining Siah1 HS in the TaC:eGFP background we observed a decrease in cones both dorsally and ventrally (Figures $5 \mathrm{E}-\mathrm{G}, \mathbf{H}$ ). The most striking decrease was again observed in the ventral portion of the retina (Figure 5G'). Having observed a negative effect of Siah1 overexpression on photoreceptor development, we next sought to determine the extent of these effects in development of other retinal cell types.

\section{Inner Retinal Neurons Are Not Affected by Elevation of Siah1 Activity}

Since Siah1 can potentially target several proteins and localizes to other regions of the retina (Figure 1), we investigated if overexpression of Siah1 could also impact the development of other cell types in the retina. Ganglion and amacrine cells are among the first retinal neurons to differentiate, a good portion having done so prior to the first HS at $48 \mathrm{hpf}$ (van Driel et al., 1990). Immunostaining of retinal sections from Siah1 HS embryos with HuC/D indicated that morphology and cell number of ganglion and amacrine cells was unaffected by Siah1 over activation (Supplementary Figures 2A-D). Similarly, bipolar cells, visualized using $\mathrm{PKC} \alpha$ immunostaining, were also found to be unaffected by Siah1 overactivation (Supplementary Figures 2E-H). Horizontal cells, visualized by Prox1 immunostaining, line the outermost part of the INL and have an oblong shape. The Prox1 + horizontal cells within the Siah1 HS embryos had normal morphology (Supplementary Figures 2E-G) but were decreased in number compared to wildtype and Siah1 $\triangle$ RING HS (Supplementary Figure 2H). This phenotype was not as severe as what we observed for rods and cones and was therefore not a focus of our investigation going forward. In summary, from our analysis of ganglion, amacrine, bipolar and horizontal cells, we concluded that the functional 

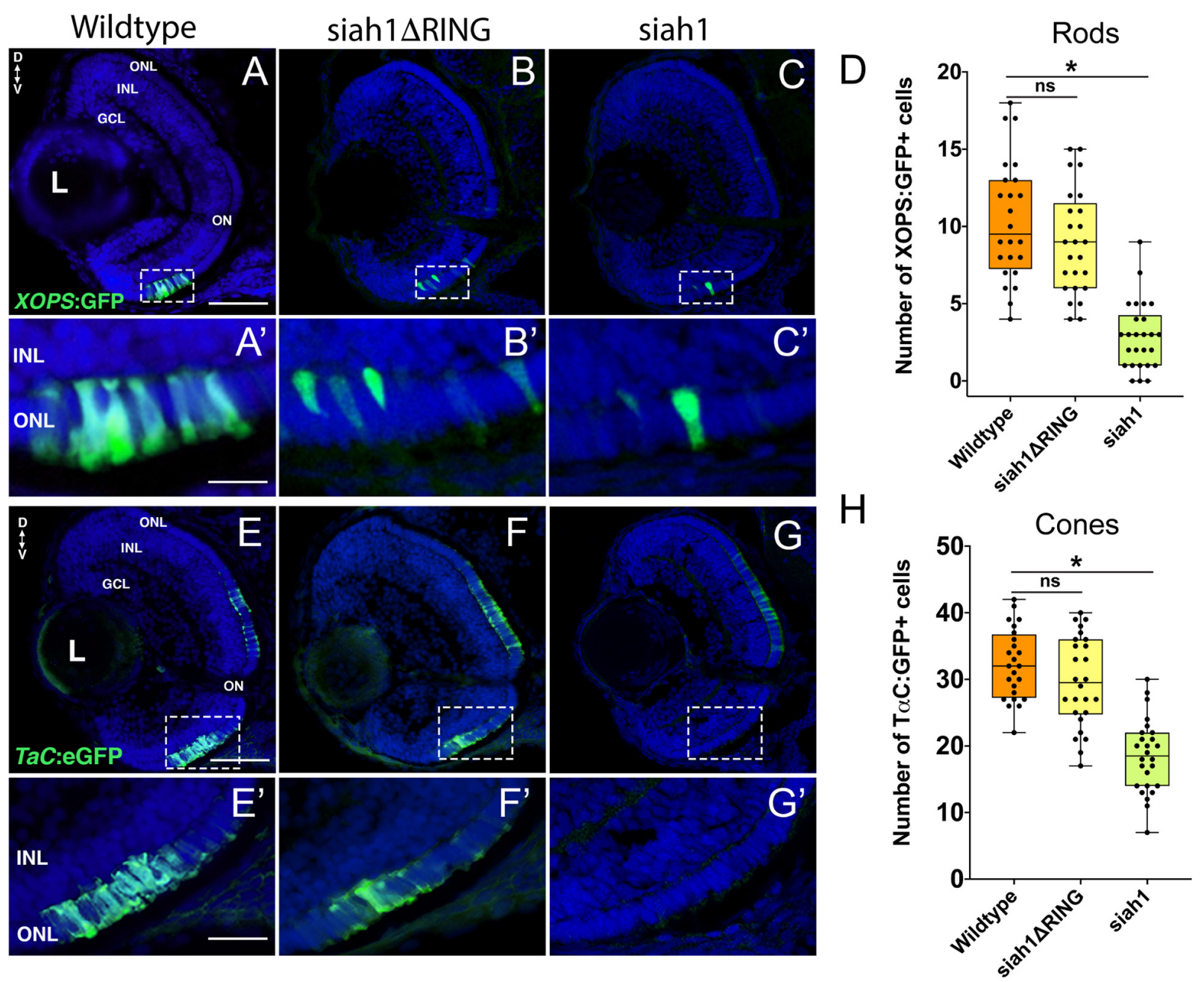

FIGURE 5 | Siah1 overexpression leads to a reduction of rod and cone photoreceptors. Retinal cryosections of Tg[XOPS:GFP] (wildtype),

Tg[hsp70:siah1]/Tg[XOPS:GFP] (siah1), and Tg[hsp70:siah1 $\Delta$ RING]/Tg[XOPS:GFP], (siah1 $\Delta$ RING) embryos were analyzed for GFP fluorescence after heat shock (HS; A-C). The number of rod photoreceptors (green) was significantly decreased in siah1 HS embryos at 72 hpf compared to wildtype and siah1 $\Delta$ RING (D). Compared to wildtype and siah1 $\Delta$ RING differentiated rod photoreceptors in Siah1 HS embryos have stunted outer segments (A'-C'). Retinal cryosections of

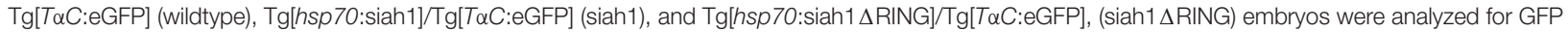
fluorescence after heat shock (HS; E-G). Compared to wildtype and siah1 $\triangle \mathrm{RING}$, siah1 HS resulted in a significant decrease in the number of cone photoreceptors (green) present the ventral portion of the retina of (E'-G',H). DNA was stained with DAPI (blue), scale bar = $50 \mu \mathrm{m}$ and $10 \mu \mathrm{m}$ (A). L, lens; ONL, outer nuclear layer; INL, Inner nuclear layer; GCL, ganglion cell layer; ON, optic nerve; D, Dorsal; and V, Ventral. Each point on the graph represents total counts from an individual embryo. ${ }^{*}=p<0.05$.

consequences of Siah1 overexpression during the 48-72 hpf stage of retinal development is mostly confined to photoreceptors. Our data suggests that high levels of Siah1 activity can specifically alter photoreceptor maturation. As such, we next began to address the potential mechanisms for how Siah1 activity impacts photoreceptor development.

\section{Siah1 Misexpression Does Not Affect Cell Proliferation}

To determine whether the decrease in rod and cone photoreceptors at $3 \mathrm{dpf}$ in Siah1 HS embryos was due to a delay in differentiation or cell death, we first assessed cell proliferation in the retina. Immunostaining for cells in $S$ phase using PCNA and cells in mitosis with PH3 was conducted to detect potential differences in cell proliferation and cell cycle progression between wildtype, Siah1 $\triangle$ RING, and Siah1 HS. Our results show no difference in PCNA + cells in the ciliary marginal zone (CMZ), an area of the retina containing stem and retinal progenitor cells that supports continuous retinal growth (Wan et al., 2016), between wildtype, Siah1 $\Delta$ RING, and Siah1 HS embryos (Supplementary Figures 3A-C). While rod photoreceptors do not come from this pool of progenitors, it is an indicator of the rate of cell proliferation in the retina. PCNA was strongly expressed across genotypes and spanned a similar area in the dorsal and ventral portion of the retina. We followed up PCNA analysis with $\mathrm{PH} 3$ immunostaining to visualize cells 
in mitosis, rather than $\mathrm{S}$ phase. While there was variation when quantifying $\mathrm{PH} 3$ + cells in all genotypes, we found no significant difference in the total number of $\mathrm{PH} 3$ + cells when comparing all of our groups (Supplementary Figure 3G). Interestingly, the distribution of the $\mathrm{PH} 3+$ cells did vary across experimental groups (Supplementary Figure $\mathbf{3 H}$ ). In the wildtype and Siah1 $\triangle$ RING HS lines, the majority of $\mathrm{PH} 3+$ cells were in the ONL (Supplementary Figures 3D,E). In contrast, in the Siah1 HS embryos, most $\mathrm{PH} 3$ + cells were found in the $\mathrm{CMZ}$ and the INL. Taken together, we conclude Siah1 does not regulate cell proliferation or cycle progression, which led us to pursue cell death as a potential mechanism for Siah1 mediated aberrant photoreceptor development.

\section{Siah1 Overexpression Results in a Proteasome-Dependent Increase in Retinal Apoptosis}

We used TUNEL staining to label apoptotic cells in the retina of all genotypes following HS. Additionally, we utilized MG132, which inhibits proteasome activity in order to assay whether the phenotypes observed are dependent on Siah1's E3 enzymatic activity (control embryos were treated with DMSO). We noted retinal cell death in the retinas of wildtype and Siah1 $\Delta$ RING HS embryos (Figures 6A,C). In wildtype and siah1 $\Delta$ RING embryos only a handful of apoptotic cells were observed, primarily located in the INL bordering the CMZ. In contrast, Siah1 HS embryos showed a significant increase in cell death when compared to wildtype and Siah1 $\triangle$ RING (Figure 6F). Apoptosis was increased in the GCL, INL, and ONL of the retina (Figure 6D). An increase in retinal apoptosis due to induction of Siah1 was first observed to occur between 60 to $66 \mathrm{hpf}$ (Supplementary Figure 5). To determine whether increased apoptosis was dependent on the proteasome we also treated heat shocked embryos with MG132. Previous work in our lab has shown that $12.5 \mu \mathrm{M}$ of MG132 is effective for embryonic inhibition of the proteasome without toxicity (Piedade et al., 2019). Cell death was significantly reduced in Siah1 HS embryos treated with MG132, bringing down the average number of TUNEL + cells to one, which was comparable to wildtype (Figures 6B,E,F). After MG132 treatment, any remaining apoptotic cells in Siah1 HS embryos were primarily located in the INL (Figure 6E). Based on these results, we suspect that reduction of cone and rod photoreceptors upon Siah1 overexpression results from increased cell death of rod and cone progenitors or immature photoreceptors. This effect appears to be dependent on the E3 ligase activity of Siah1 as proteasome inhibition was able to rescue the phenotype. Based on these findings, we next investigated whether Siah1targeting of Cdhrla contributes to the photoreceptor phenotype of Siah1 overexpressing retinas.

\section{Siah1 Targeting of Cdhr1a for Proteasomal Degradation Regulates Photoreceptor Development and Survival}

Having shown that MG132 could rescue Siah1 induced apoptosis in the retina, we next examined whether inhibition of apoptosis by MG132 would also rescue photoreceptor development.
Treating Siah1 HS embryos with MG132 for $24 \mathrm{~h}$ not only decreased cell death throughout the retina, but also rescued the number of mature and immature rod photoreceptors (Figures 6G-K). Quantification of immature and mature rods, visualized by 4C12 immunostaining (Morris et al., 2005), indicated a significant increase in the number of rod cells upon MG132 treatment (Figure 6L). Mature and immature rod photoreceptors were present in the dorsal and ventral portion of the retina in Siah1 HS MG132 treated embryos (Figure 6K). The average number of rod photoreceptors was slightly lower in the Siah1 HS MG132 treated embryos compared to wildtype but increased by over 50\% when compared to Siah1 HS DMSO treated embryos (Figure 6L). We observed similar results when examining consequences of MG132 treatment in Tg[XOPS:GFP]/Tg[hsp70:siah1] HS embryos (Supplementary Figures 4A-E). Importantly, cone photoreceptor numbers were also increased upon MG132 treatment, as observed in $\operatorname{Tg}[T \alpha C:$ GGFP $] / \operatorname{Tg}[$ hsp70:siah1] HS embryos (Supplementary Figures 4F-J). Quantification of cone photoreceptors in these embryos showed MG132 treatment led to significantly more cells compared to DMSO treatment (Supplementary Figure 4J). We therefore conclude that excess Siah1 E3 ligase activity likely leads to increased apoptosis in the ONL and may drive the reduction of both rod and cone photoreceptors.

Having shown that Siah1 and Cdhrla can interact in cell culture, and that Siah1 can regulate Cdhrla levels, we next examined whether Siah1 targeting of Cdhrla was responsible for the observed photoreceptor phenotypes. As outlined previously, CDHR1 is known to have an established role in photoreceptor maintenance, in particular turnover of outer segment disks (Burgoyne et al., 2015). What is less clear is whether CDHR1 plays a role during development of photoreceptors. As such, we hypothesized that Siah1 targets Cdhrla and the reduction in Cdhrla protein levels leads to apoptosis and subsequent reduction of photoreceptor progenitor cells. To test this hypothesis, we injected single cell stage $\operatorname{Tg}[h s p 70$ :siah1] embryos with wildtype (WT) cdhrla mRNA, performed our HS treatment and analyzed rod and cone photoreceptors at $72 \mathrm{hpf}$. Injection of mRNA had no observable effect on WT or Siah1 $\triangle$ RING HS embryos, however, in Siah1 HS embryos we observed a significant increase in the number of both rod and cone photoreceptors compared to Siah1 HS alone at $72 \mathrm{hpf}$ (Figures 7, 8). Both mature and immature rod cells were increased in number when Siah1 HS embryos were injected with WT cdhrla mRNA (Figures 7A-E). Similar outcomes were observed when using Tg[XOPS:GFP]/Tg[hsp70:siah1] embryos (Figures $7 \mathbf{F}-\mathbf{J}$ ). Not only were the number of cells increased, but the rod cells appeared elongated, contained outer segments and were evenly spaced. When examining cone cells using the $\operatorname{Tg}[\mathrm{TaC}: \mathrm{eGFP}] / \mathrm{Tg}[h s p 70: \operatorname{siah} 1]$ we also documented that injection of WT cdhrla mRNA rescued the number of cone cells to levels comparable to wildtype or siah1 $\triangle \mathrm{RING}$ HS embryos (Figure 8). Our results show that an excess of Cdhrla can overcome the Siah1-mediated targeting for proteasomal degradation and therefore protect development of photoreceptor cells. 


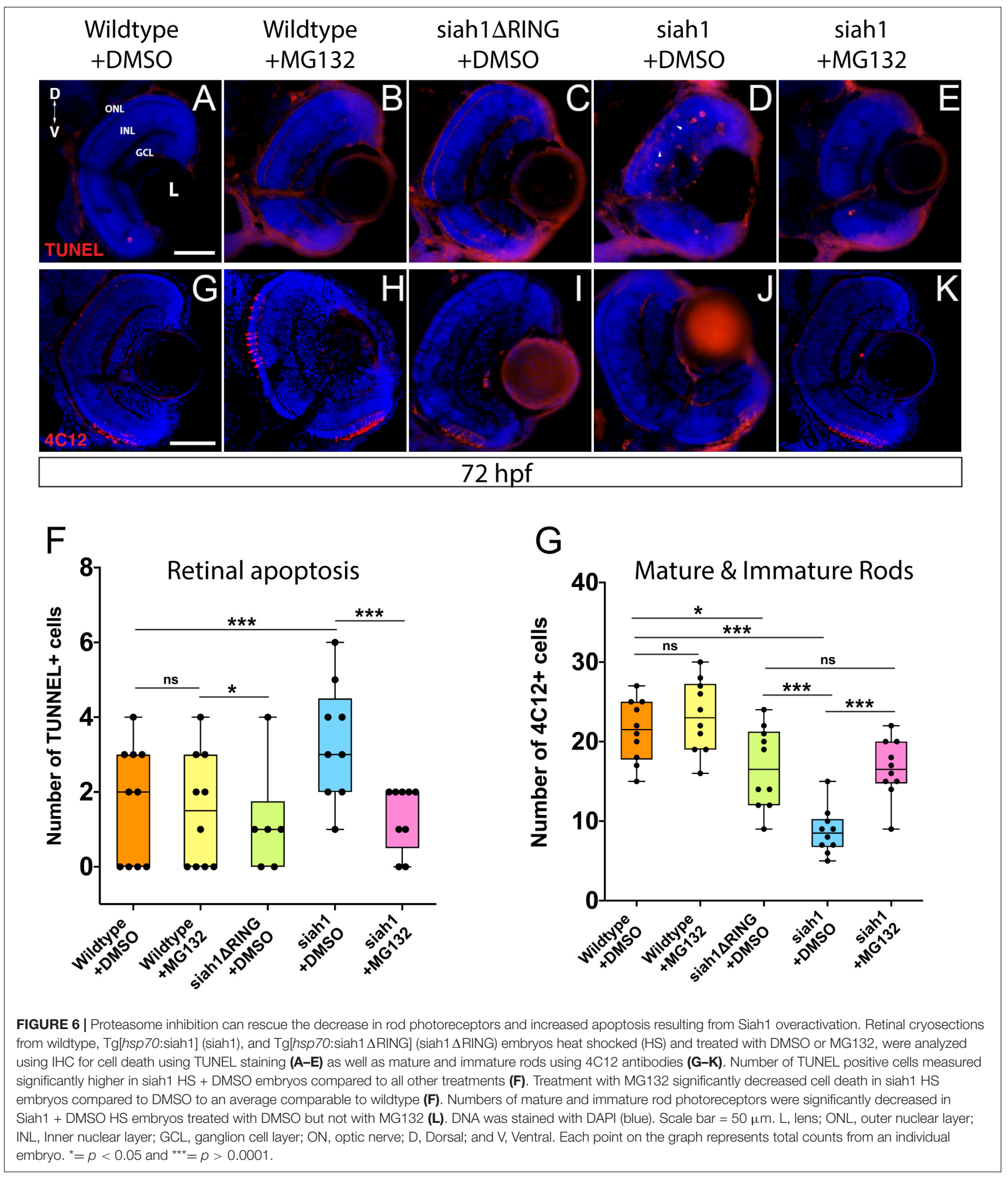

To further extend our analysis, we also attempted rescue of the Siah1 HS phenotype using the Cdhrla degron mutant construct, $c d h r 1 a^{\mathrm{LmA}}$. Based on the insensitivity of Cdhrla ${ }^{\mathrm{LmA}}$ to Siah1 activity we predicted it would have an enhanced rescue effect. Single cell embryos were injected with $c d h r 1 a^{\mathrm{LmA}}$ mRNA and subjected to our HS protocol. Strikingly, $c d h r 1 a^{\mathrm{LmA}}$ was more efficient at protecting rod (Figures $\mathbf{7} \mathbf{C}, \mathbf{G}$ ) and cone cells (Figures 8A-D) from the effects of Siah1 overexpression than 

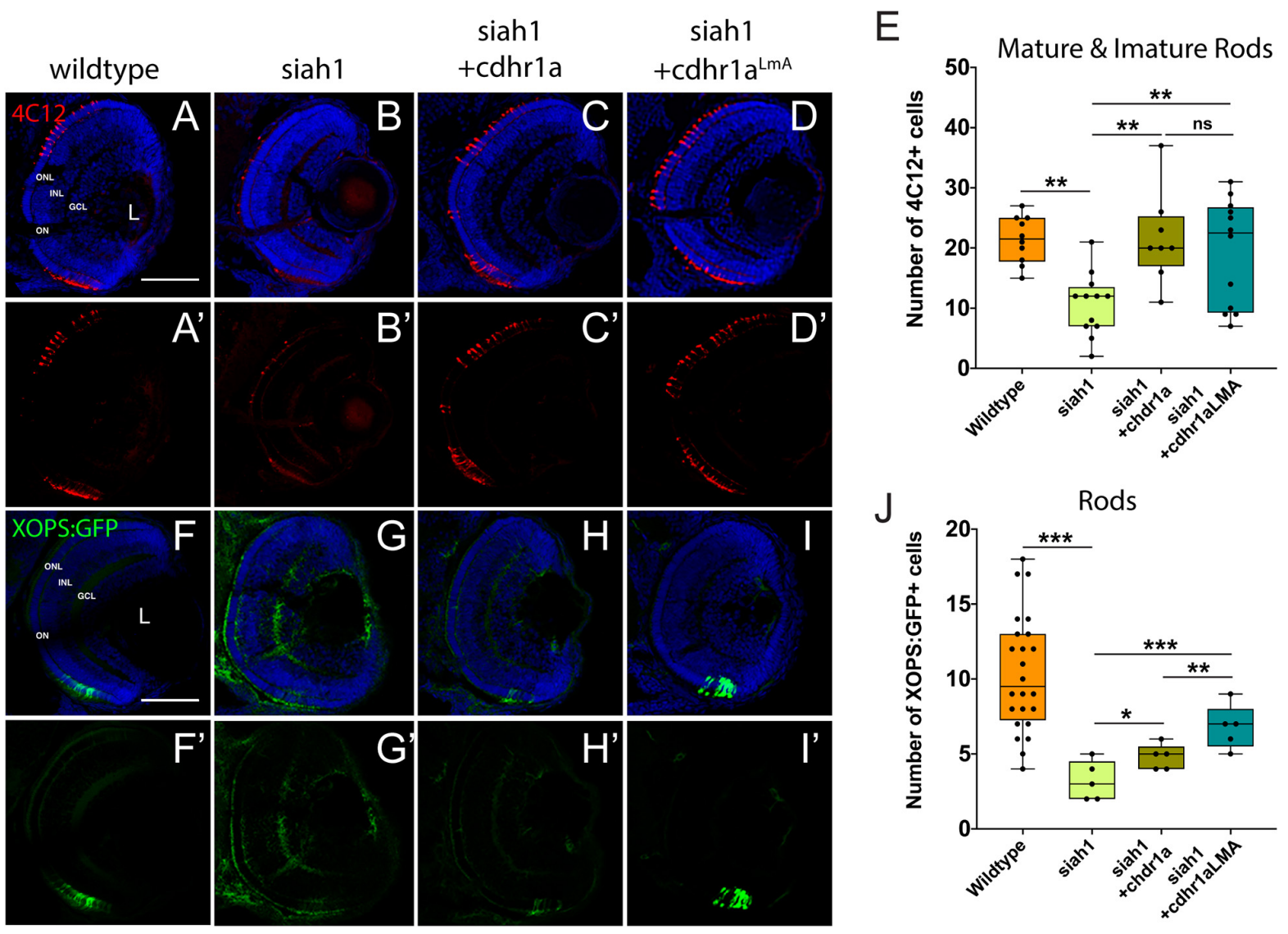

FIGURE 7 | Rod photoreceptor development relies on sufficient levels of Cdhr1a. Retinal cryosections from wildtype or Tg[hsp70:siah1]/Tg[XOPS:GFP] (siah1), injected with wild type cahr1a or cdhr1a ${ }^{\mathrm{LMA}}$ mRNA were heat shocked (HS) and analyzed for immature and mature rod cells using IHC for 4C12 (red; A-D'). Injection of both cdhr1a and cdhr1a ${ }^{\mathrm{LMA}}$ mRNA increased the number of immature and mature rod cells compared to siah1 HS alone (E). Retinal cryosections from Tg[hsp70:siah1]/Tg[XOPS:GFP] (siah1), injected with wildtype cdhr1a or cdhr1a LMA mRNA were heat shocked (HS) and analyzed for GFP signal (green; F-I'). Injection of both cdhr1a and cdhr1a ${ }^{\mathrm{LMA}}$ mRNA increased the number of GFP + rod cells compared to siah1 HS alone, with cdhr1a ${ }^{\mathrm{LMA}}$ giving a significantly stronger response (J). DNA was stained with DAPI (blue). Scale bar = 50 $\mu \mathrm{m}$. L, lens; ONL, outer nuclear layer; INL, Inner nuclear layer; GCL, ganglion cell layer; and ON, optic nerve. Each point on the graph represents total counts from an individual embryo. ${ }^{*}=p<0.05,{ }^{* *}=p>0.001$, and ${ }^{* * *}=p>0.0001$.

WT $c d h r 1 a$. In particular, injection of $c d h r 1 a^{\mathrm{LmA}}$ resulted in significantly more XOPS:GFP + mature rod photoreceptor cells compared to WT cdhrla (Figures 7D,H). Both WT and LmA were able to rescue the number of $\mathrm{TaC}$ :eGFP positive cones, but with LmA having a much tighter distribution (Figure 8E). Taken together, we show that deficiencies in rod and cone photoreceptor development upon induction of Siah1 activity correlate with levels of Cdhrla protein. We therefore conclude that Siah1mediated regulation of Cdhrla protein levels is important during photoreceptor development.

Finally, to directly ascertain a functional role for Cdhrla during photoreceptor development, we analyzed rod and cone status upon inactivation of Cdhrla using CRISPR/Cas9. In order to achieve high efficiency cutting in the F0 generation we injected two Alt-R-CRISPR crRNAs targeting adjacent regions of cdhrla coding sequence. Genomic PCR analysis indicated a cutting success rate of $75 \%+$ in our injected embryos (Supplementary Figure 6). Cdhrla crRNAs were injected into Tg[XOPS:GFP] or $\operatorname{Tg}[T \alpha C: \mathrm{eGFP}]$ embryos, grown up to $72 \mathrm{hpf}$, screened for
CRISPR cutting and analyzed for rod and cone phenotypes (Figure 9). Embryos predicted to harbor insertions or deletions were analyzed for cdhrla levels in the ONL via IHC. As expected, we failed to detect Cdhrla signal in the ONL of embryos injected with cdhrla crRNAs while seeing no effect in embryos injected with control Golden crRNA (Figure 9A). Similar to Siah1 over activation, CRISPR-induced loss of Cdhrla function led to a significant decrease in cone and rod cells (Figures 9B-E). This result further supports our findings that Cdhrla is necessary for rod and cone development and/or survival and that Siah enzymes may regulate this process.

\section{DISCUSSION}

Several studies in the past decade have associated mutations in the human CDHR1 gene with cone-rod dystrophies (Bolz et al., 2005; Henderson et al., 2010; Ostergaard et al., 2010; Cohen et al., 2012; Duncan et al., 2012; Ba-Abbad et al., 2013; 


\section{Wild type HS}

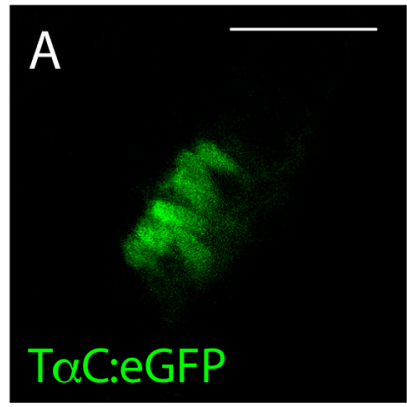

Siah1 HS

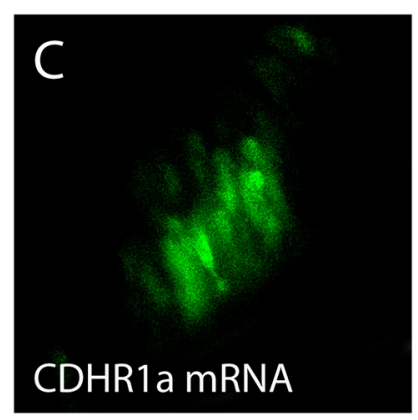

Siah1 HS

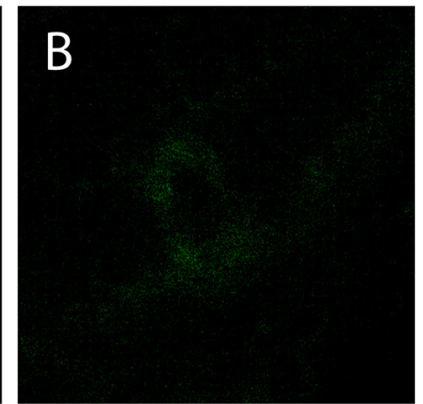

Siah1 HS

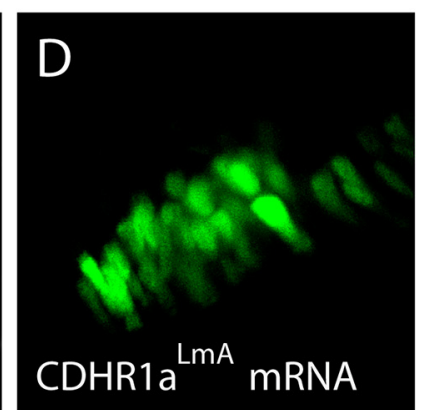

E

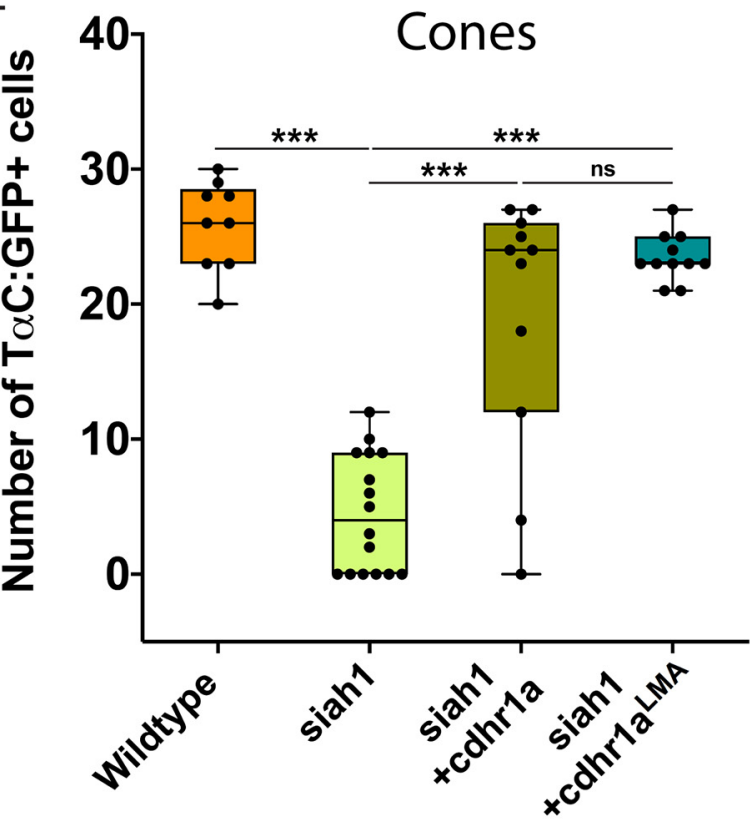

FIGURE 8 | Cone photoreceptor development relies on sufficient levels of Cdhr1a. Confocal stacks of heat shocked (HS) Tg[TaC:eGFP] (wildtype) and Tg[hsp70:siah1]/Tg[TaC:eGFP] (siah1) embryos or those injected with cdhr1a mRNA or cdhr1a ${ }^{\mathrm{LMA}}$ mRNA were analyzed in 3D for GFP fluorescence. Quantification was restricted to the ventral retina (A-D). Injection of both wildtype and the LMA variant of cdhr1a mRNA resulted in numbers of GFP + cone cells comparable to that of wildtype (E). Scale bar $=50 \mu \mathrm{m} .{ }^{* \star *}=p<0.0001$.

Bessenyei and Oláh, 2014; Nikopoulos et al., 2015; Arno et al., 2016; Riera et al., 2017; Stingl et al., 2017; Fu et al., 2018). While the mechanism as to how loss of CDHR1 function affects pathogenesis of cone-rod dystrophies is unknown, several studies have reinforced its importance to photoreceptor cell biology by characterizing its protein localization (Rattner et al., 2001, 2004; Burgoyne et al., 2015) and necessity for photoreceptor disk renewal (Ostergaard et al., 2010). In particular, it has been shown that CDHR1 links immature disks to the inner segment prior to their incorporation into the outer segment (Burgoyne et al., 2015). However, none of the current studies examined in detail whether CDHR1 had any involvement in vertebrate photoreceptor cell development. This is of particular note, especially when considering possible inheritable associations with cone-rod dystrophy predisposition. In this study we describe a post-translational modification mechanism, controlled by the Siah1 E3 ubiquitin ligase, which regulates the stability of Cdhrla to mediate vertebrate photoreceptor cell maturation, and survival. Our work outlines the first observation of a functional role for Siah1 and Cdhrla during photoreceptor development, which may in future studies be utilized to examine the mechanism of cone-rod dystrophy pathogenesis.

Cdhrl encodes a photoreceptor cell specific cadherin; a single-pass transmembrane glycoprotein with calciumdependent adhesive abilities as well as signaling functions. Cadherin extracellular domains contain several tandem repeats of negatively charged amino acids which are responsible for interaction with extracellular molecules including other cadherins (Babb et al., 2005; Pontoriero et al., 2009; Clendenon et al., 2012). During eye development, cadherins have been implicated in the separation of the invaginated lens vesicle from the surface ectoderm (Pontoriero et al., 2009), initiation and elongation of the RGC axons and dendrites (Masai et al., 2000; Lele et al., 2002; Malicki et al., 2003; Babb et al., 2005), differentiation of RGC and amacrine cells (Babb et al., 2005; Clendenon et al., 2012), activation of proliferation in the eye primordium (Pujic and Malicki, 2001; Malicki et al., 2003; Babb et al., 2005), and retinotectal axon projection (Clendenon et al., 2012). In line with previous studies (Rattner et al., 2001, 2004; Burgoyne et al., 2015), we confirmed cdhrla gene expression and protein localization to be specifically in the base of the outer segment of zebrafish rod and cone photoreceptor cells. Zebrafish Cdhrla protein localized to a narrow stalk region, known as the connecting cilium of the photoreceptor cell. The connecting cilium bridges the outer segment with the cell body and is critical for proper trafficking of proteins, like rhodopsin, from the cell body to the outer segment (Eblimit et al., 2018). This region is also the site of new disk assembly and release during maintenance of rod outer segments. Mutations in structural proteins of this region are known to associate with juvenile Retinitis Pigmentosa (Eblimit et al., 2018), highlighting its relevance in photoreceptor cell development. Furthermore, 
A

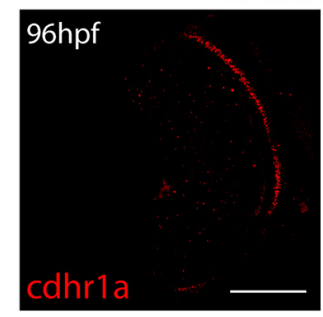

\section{Golden CRISPR}

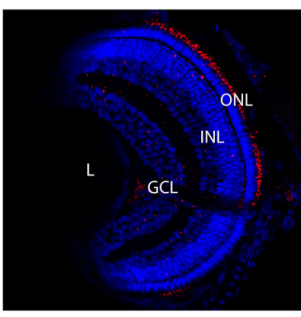

\section{cdhr1a CRISPR}

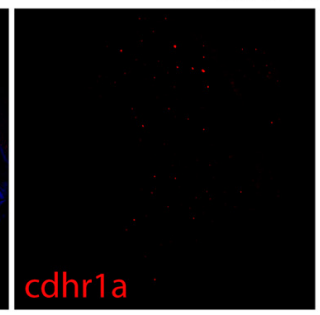

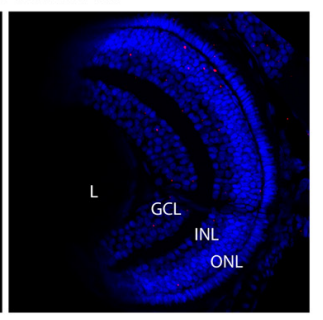

B
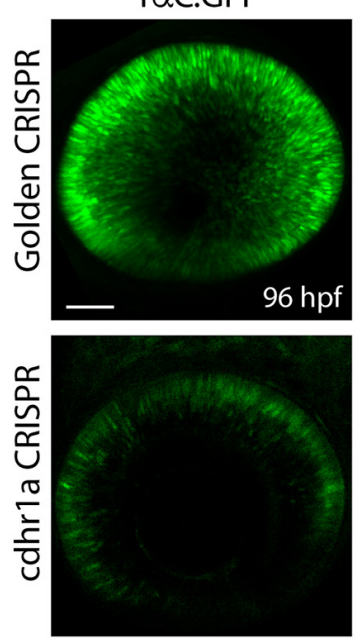

Whole retina
C

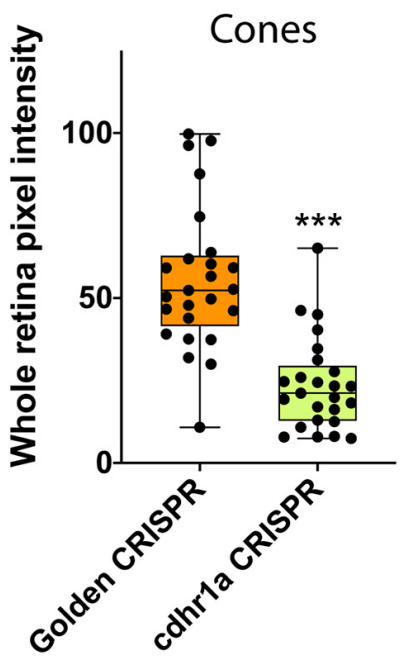

D

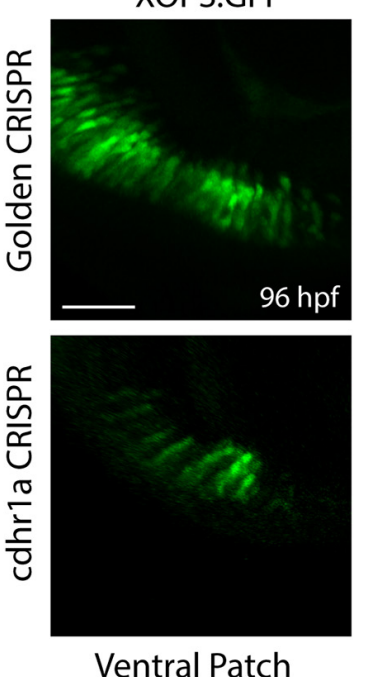

E

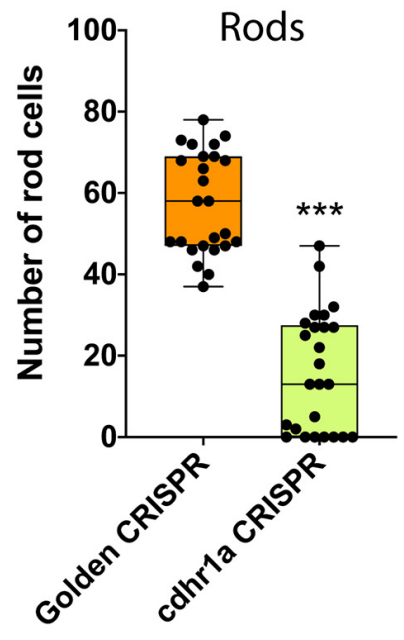

FIGURE 9 | CRISPR-induced loss of Cdhr1a function hinders rod and cone cell development. Cdhr1a protein localization (red) was determined using IHC in retinal cryosections from $4 \mathrm{dpf}$ old Golden crRNA or cdhr1a crRNA injected embryos (A). Confocal stacks of Alt-R-CRISPR crRNA injected Tg[TaC:eGFP] embryos analyzed in 3D for GFP fluorescence at 96 hpf. Golden crRNA, targeting non-coding sequence is used as a negative control (B). Quantification of whole retina fluorescence intensity normalized to retinal area. Each point on the graph represents an individual embryo (C). Confocal stacks of Alt-R-CRISPR crRNA injected Tg[XOPS:GFP] embryos analyzed in 3D for GFP fluorescence at 4 dpf. Golden crRNA, targeting non-coding sequence is used as a negative control (D). Quantification of XOPS:GFP positive rod cells in the ventral retina. Each point on the graph represents an individual embryo (E). DNA was stained with DAPI (blue). Scale bar = $50 \mu \mathrm{m}$. L, lens; ONL, outer nuclear layer; INL, Inner nuclear layer; and GCL, ganglion cell layer. ${ }^{\star \star *}=p<0.0001$.

cadherins, through their cytoplasmic domains are able to link with the cytoskeleton by interacting with catenins (Pontoriero et al., 2009). These interactions are responsible for maintaining polarization of the highly stratified epithelial tissues, such as the retina (Clendenon et al., 2012). When cells of epithelial tissues have blocked their ability to maintain cellular adhesion with the surrounding cells or the extracellular matrix by the loss of cadherin function, for example, they undergo a process of apoptosis called anoikis (Pontoriero et al., 2009).

Our observation that zebrafish Cdhrla protein localizes to the connecting cilium as soon as photoreceptor cells are formed reinforces the notion of CDHR1's importance to photoreceptor biology. However, we lacked an understanding of its regulation. Our FWISH results indicated that expression of siah1 and siah $2 l$ co-localizes with cdhrla in the ONL from 3 and up to $5 \mathrm{dpf}$ of the zebrafish retina. Furthermore, using a cell culture model, we were able to demonstrate direct interaction, via co-IP experiments, and showed that Siah1 targets Cdhrla for proteasomal degradation. In addition, we show that inactivation of the Siah1 E3 domain (siah1 $\Delta$ RING), proteasomal inhibition (MG132), or mutation of the Siah1 degron motif $\left(c d h r 1 a^{\mathrm{LmA}}\right)$ prevents Cdhrla degradation. Taken together, our data strongly supports that Cdhrla is a direct target for Siah1 and that both are expressed in the same place and at the same time.

To examine the consequences of Siah1-mediated regulation of Cdhrla stability during photoreceptor development we used a HS mediated overexpression approach. This enabled us to control the timing and extent of Siah1 overactivation. In particular we wanted to avoid interfering with early embryonic development so as to prevent non-specific phenotypes. Siah1 overexpression resulted in a significant decrease in the amount of rod and cone photoreceptor cells. All other retinal cell types were unaffected by Siah1 overexpression. The reduction in rods and cones coincided with a significant increase in TUNEL + apoptotic cells in the retina, and in particular in the ONL (Figure 6D). As expected, inhibition of proteasome activity rescued the Siah1 overactivation phenotype. The cell death we noted could result from cellular loss of contact with the extracellular matrix and/or neighboring cells 
mediated by Cdhrla in photoreceptor precursor cells. Recently, a mouse conditional double knockout for $\mathrm{E}$ and $\mathrm{N}$-cadherin had increased number of TUNEL-positive cells in the lens (Pontoriero et al., 2009). Cell death was also noted in the retina of cdh11 and cdh4 morphants (Babb et al., 2005; Clendenon et al., 2012). Interestingly, when we injected WT or the LmA Siah insensitive cdhrla mRNA and overactivated Siah1, rod and cone photoreceptor numbers were rescued. We therefore propose that Cdhrla stability in the presence of excess Siah1 is critical for photoreceptor cell survival during development. TUNEL positive cells were observed not only in the ONL, but also in other retinal regions. This may represent apoptosis of progenitor cells that are in the process of migrating to the ONL. The fact that overactivation of Siah1, which will lead to a decrease in Cdhrla protein levels, leads to increased apoptosis and ultimately significant reduction in rods and cones partially supports this hypothesis. In addition, results from Alt-R-CRISPR crRNA injections, which have been shown to be highly efficient in generating biallelic indel mutations and therefore enable examination of phenotypes in F0s, indicate that loss of Cdhrla function also leads to a decrease in rods and cones (Figure 9).

When comparing our results to those of previous studies (Rattner et al., 2001, 2004; Burgoyne et al., 2015), we propose three potential roles for Cdhrla during photoreceptor development. First, based on its cadherin function, Cdhrla may be required for the organization of cytoskeletal elements at the base of newly forming outer segments. In its absence, failure of outer segment formation may trigger apoptosis and subsequent reduction in photoreceptor cells. Second, Cdhrla, via its extracellular domains, could interact with extracellular matrix in the ONL. In this proposed role, Cdhrla contributes to either photoreceptor precursor migration and targeting or subsequent photoreceptor adhesion required for survival. The absence of Cdhrla function could therefore either reduce the number of photoreceptor precursors reaching the ONL leading to reduction of mature rods and cones or may affect maturing rod and cone survival due to absent or improper cell-cell adhesion. Both of our hypotheses are supported by results from our Siah overexpression experiments, which lead to a reduction in Cdhrla function. Furthermore, our model finds clear support from the transcriptomic analysis recently employed by Kaewkhaw et al. (2015) showing increased levels of CDHR1 in photoreceptor progenitor cells during differentiation in $3 \mathrm{D}$ human retina cultures. Lastly, based on its localization, Cdhrla is predicted to regulate the release of newly formed outer segment disks to ensure proper function of rods and cones (Burgoyne et al., 2015). One could imagine that assembly of the very first disks would also require Cdhrla function and in its absence this process might fail and lead to apoptosis. Upon decrease of Cdhrla levels, the connection between the innermost outer segment disks and the inner segment of the photoreceptor could weaken, preventing outer segment disk formation and eventual death of the photoreceptor prior to maturity (Figure 8). Interestingly, it currently remains unknown as to how Cdhrla releases the disks. It may be possible that Siah1 targets Cdhrla for degradation and this regulates the timely release of the disks. Investigating Siah1 function in juvenile and adult photoreceptors will need to be performed to assess these possibilities.

In conclusion, we provide the first direct evidence that Cdhrla plays a critical role during photoreceptor development, maturation, and survival. Furthermore, we show that Cdhrla is directly regulated by the UPS via interaction with Siah1. Our findings have new implications for examination of Cdhrlaassociated cone-rod dystrophy as well as the role of UPS during photoreceptor development. Future studies will focus on the exact mechanism of Cdhrla function in both photoreceptor progenitors as well as immature rods and cones. Furthermore, it will be important to assess the role of Cdrh1a and Siah1 during retinal regeneration and adult photoreceptor outer segment maintenance. Understanding these mechanisms will be imperative to identifying therapeutic strategies for the growing population of individuals suffering from sight-threatening diseases such as cone-rod dystrophy.

\section{MATERIALS AND METHODS}

\section{Zebrafish Husbandry and Embryo Maintenance}

Zebrafish husbandry used in all procedures were approved by the University of Kentucky Biosafety office as well as IACUC Policies, Procedures, and Guidelines (IACUC protocol 20151380). The AB strain was used as wildtype. Transgenic lines used to visualize rod and cone photoreceptors were: Tg[XlRho:EGFP] (XOPS:GFP; Fadool, 2003) and Tg[3.2TaC:eGFP] (TaC:eGFP; Kennedy et al., 2007), respectively. Embryos were kept at $28^{\circ} \mathrm{C}$ in E3 embryo media. At indicated times, embryos or larvae were anesthetized in tricaine and fixed with $4 \%$ PFA in PBS overnight at $4^{\circ} \mathrm{C}$.

\section{Fluorescent Whole-Mount in situ Hybridization}

Fluorescence whole-mount in situ hybridization was performed as a modification from previously described (Lauter et al., 2011). Embryos were permeabilized with $10 \mathrm{mg} / \mathrm{mL}$ Proteinase $\mathrm{K}$ for $30 \mathrm{~min}$ for $3 \mathrm{dpf}$ embryos, $60 \mathrm{~min}$ for $4 \mathrm{dpf}$ embryos, and 75 min for 5 dpf larvae. Digoxigenin (DIG) and fluorescein labeled (FITC) probes were synthesized by using DIG and FITC RNA labeling kit (Roche). Primer sequences are listed in Supplementary Table 1. Anti-Digoxigenin-AP, Fab fragment (ROCHE) and anti-fluorescein-AP, Fab fragment (Roche) antibodies, Fast blue (SIGMA) and Fast-red (SIGMA) were used to detect the hybridization signal. 20-30 embryos were analyzed at each timepoint in 2-3 independent experiments. Images presented are representative of the results observed.

\section{Cryosectioning and Cell Counts}

Embryos were fixed in $4 \%$ paraformaldehyde then washed overnight in $10 \%$ then $30 \%$ sucrose overnight at $4^{\circ} \mathrm{C}$. Transverse, $10 \mu \mathrm{m}$, sections collected, beginning just anterior to and ending posterior to the eye. For imaging and cell quantification, sections containing an optic nerve were used for consistency. All 
photoreceptors in the dorsal, central, and ventral portions of the retinal ONL were quantified. Retinal size did not vary between conditions and was therefore not used for normalization. For the $\mathrm{HuC} / \mathrm{D}, \mathrm{PKC} \alpha$, and Proxl quantification, counts were conducted on $50 \mu \mathrm{m}$ wide regions of interest, $50 \mu \mathrm{m}$ dorsal to the optic nerve for consistency. Images presented are representative of the results observed.

\section{Heat Shock Inducible Transgenic Zebrafish Lines}

Tg[hsp70:Siah1], Tg[hsp70:Siah1 $\Delta$ RING], were generated by amplification the full coding region (Siah 1: Ensembl transcript ID: ENSDART00000026679.8) from 72 hpf zebrafish cDNA. The dominant negative Siah1 construct, Siah1 $\Delta$ RING, was previously described (Piedade et al., 2019). Both constructs were amplified with primers (Supplementary Table 1) containing attB for Gateway cloning into pDONR221 using BP Clonase II (Invitrogen). pDONR221 clones were verified by sanger sequencing (eurofins). Using Gateway LR Clonase II, according to the manufacturer's protocol, (Invitrogen), pDEST constructs pDestTol2CGred (red heart marker; gift from Dr. Allison, University of Alberta) were combined with pDONR221 plasmids, the 5 ' element HS promoter plasmid (p5E-hsp70) and the 3' element (p3E-polyA) plasmid. Positive clones were verified by sanger sequencing (eurofins). Verified constructs were injected (50 pg) along with Tol 2 mRNA (100 pg) and dextran-red into zebrafish zygotes. Transgenic founder embryos were screened at 48-72 hpf for heart marker fluorescence. Founders were outcrossed to wild-type and screened for germline transmission to create the F1 generation.

\section{Heat Shock Experiment Design}

For HS at all developmental stages, groups of 50 embryos were incubated at $38^{\circ} \mathrm{C}$ for $30 \mathrm{~min}$ using a recirculating water bath. Embryos were then removed from the water bath and placed back in the $28^{\circ} \mathrm{C}$ incubator in fresh $\mathrm{EB}$ media according to the time schedule outlined in Figure 4B. In short, embryos were shocked twice, once at $48 \mathrm{hpf}$ and once at $60 \mathrm{hpf}$.

\section{Immunohistochemistry and TUNEL Assay}

Immunohistochemistry was conducted as previously described (Coomer and Morris, 2018) and imaged on a confocal microscope (Leica SP8, Leica). The following antibodies were used: anti-zCDHR1a (CDHR1a, rabbit, 1:100, Bosterbio, Pleasonton, CA, United States), anti-zSiah1 (Siah1, rabbit, 1:100, Bosterbio, Pleasonton, CA, United States), anti-Huc/D (ganglion and amacrine cells, mouse, 1:40), anti-PKC $\alpha$ (bipolar cells, mouse, 1:100, Santa Cruz Biotechnology, Dallas, TX, United States), anti-Prox1 (horizontal cells, rabbit, 1:1000, Acris, San Diego, CA, United States), anti-PCNA (cells in S-phase, mouse, 1:100, Santa Cruz Biotechnology, Dallas, TX, United States), and activated caspase 3 (apoptotic cells). Alexa fluor conjugated secondary antibodies (Invitrogen, Grand Island, NY, United States) and Cy-conjugated secondary antibodies (Jackson ImmunoResearch, West Grove, PA) were used at 1:200 dilution and DAPI to label nuclei (1:10,000, Sigma, St. Louis, MO, United States). TUNEL assay was conducted with ApopTag Fluorescin Direct In Situ Apoptosis Detection Kit (Millipore, Billerica, MA, United States) on retinal cryosections according to manufacturer's instructions.

\section{MG132 Treatment}

Thirty embryos were transferred at $52 \mathrm{hpf}$ ( $3 \mathrm{~h}$ post heat shock) into a $35 \mathrm{~mm}$ petri dish containing $5 \mathrm{~mL}$ E3 embryo media plus $12.5 \mu \mathrm{M}$ MG132 (Sigma-Aldrich), or an equal volume of vehicle (DMSO, $\sim 0.01 \%$ ) until $72 \mathrm{hpf}$. The treatment was refreshed at $61 \mathrm{hpf}$, immediately after the second HS. At $72 \mathrm{hpf}$, embryos were anesthetized in tricaine and fixed with $4 \%$ PFA in PBS overnight at $4^{\circ} \mathrm{C}$ and washed with $\mathrm{PBS}$ with $0.5 \%$ Tween-20 (PBS-T) 3 times for $10 \mathrm{~min}$.

\section{DNA Constructs, mRNA Synthesis and Microinjections}

All primers used are catalogued in Supplementary Table 1. Full coding domain sequences for CDHR1a (Ensembl transcript ID: ENSDART00000026679.8) were amplified and cloned into PCS2+. Cdhrla ${ }^{\mathrm{LMA}}$ was generated by site directed mutagenesis of the WT cdhrla construct and verified by sequencing (eurofins), then cloned into pCS2+. pCS2-CDHR1a and CDHR1a ${ }^{\text {LMA }}$ plasmids were linearized with NotI (NEB). mRNA was synthesized using SP6 mMessage mMachine kit (Ambion) and purified using YM-50 Microcon columns (Amicon, Millipore). mRNA concentration was quantified using spectrophotometry. The mRNA was diluted using nuclease-free water and embryos were injected into the yolk of the embryo at single-cell stage. $100 \mathrm{pg}$ of mRNA was used as indicated in the results section.

\section{Transfection, Co-immunoprecipitation and Western Blotting}

For HEK 293 cells transfections, full coding domain sequences for Siah1, Siah1 $\triangle$ RING, CDHR1a, and CDHR1a LMA were amplified and cloned into pCIG2 using In-Fusion HD cloning Plus (Takara). Primers for Siah1 and Siah1DRING included a MYC tag while primers for CDHR1a and CDHR1a ${ }^{\text {LMA }}$ included a single FLAG tag. All constructs were verified using sanger sequencing. The HEK 293 cells were cultured at $37^{\circ} \mathrm{C}$ in DMEM media until they $80 \%$ confluency and transfected using TransITLT1 Transfection Reagent (Mirus) at $37^{\circ} \mathrm{C}$ for $24 \mathrm{~h}$. Where indicated, treatment with $10 \mu \mathrm{M}$ of MG132 for the last $4 \mathrm{~h}$ of transfection was performed. Co-IP was performed using lysates from cells treated with MG132 for $6 \mathrm{~h}$ prior to lysis. Western blotting and co-IP were performed as previously described (Piedade et al., 2019).

\section{Microscopy and Quantification}

For the FWISH, embryos were mounted in 1\% low-melting agarose in a glass bottom fluorodish (World Precision Instruments) prior to imaging using Nikon C2 confocal under the 20X (0.95 na) objective. For IHC, the slides were mounted in $40 \%$ glycerol (PBS) with coverslips and then imaged 
using either a Nikon C2 confocal under the 20X (0.95 na) and 60X (1.4 na) objectives or Leica SP8 confocal under the 20x (0.7 na) objective. Images were adjusted for contrast and brightness using Adobe Photoshop. Quantification of XOPS:GFP and TaC:eGFP positive cells was performed from 3D stacks of specifically the ventral retina using a standardized area of interest that included all the cells in the region. Stacks were collected at $3 \mu \mathrm{m}$ intervals to generate a volume that encompasses all of the fluorescent signal. 3D rotation software (Elements, Nikon) was used render the images and quantify the number of cells in the volume. Images presented are representative of the results observed.

\section{Statistical Analysis}

Two-factor analysis was done by Unpaired Students $t$-test using GraphPad $^{1}$. Data are shown as mean \pm St. dev. By conventional criteria, a $P$ value of less than 0.05 was considered significant. ANOVA analysis was performed using Prism8. ${ }^{*} p<0.05$, ${ }^{* *} p<0.001$, and ${ }^{* * *} p<0.0001$.

\section{Alt-R-crRNA CRISPR Injections}

Alt-R cdhrla and golden crRNA, tracrRNA was pre-designed and synthesized by IDT. crRNAs used were: Dr.Cas9.CDHR1A.1.AB and Dr.Cas9.CHDR1A.1.AE. Golden crRNA was custom synthesized by IDT according to sequence from Hoshijima et al. (2019). Duplex formation and dilution with Alt-R Cas9 v.3 enzyme was carried out as described by Hoshijima et al. (2019).

\section{DATA AVAILABILITY STATEMENT}

The datasets presented in this study can be found in online repositories. The names of the repository/repositories and accession number(s) can be found in the article/Supplementary Material.

\section{ETHICS STATEMENT}

The use of zebrafish in this study was approved by the University of Kentucky IACUC committee, Institutional PHS Assurance \#D16-00217 (A3336-01) with a protocol number: 2015-1370. All experimental protocols were approved by the University of Kentucky Institutional Biosafety Committee, registration number B18-3186-M.

\section{AUTHOR CONTRIBUTIONS}

WP and KT-T designed and carried out all of the experiments. WP, KT-T, and JKF were involved data analysis. JKF and ACM obtained funding and supervised the project. All authors were involved in writing and editing the manuscript.

${ }^{1}$ https://www.graphpad.com

\section{FUNDING}

This work was supported by grants from the National Institutes of Health (R01EY021769, to ACM and EY02780501 to JKF). WP was supported by Brazilian National Council for Scientific and Technological Development (CNPq) under grant number 202970/2014-0 and the Morgan Fellowship from the department of Biology, University of Kentucky. KT-T was supported by the Lyman T Johnson scholarship and department of Biology Merit Fellowship, University of Kentucky. This project was supported by the department of Biology, University of Kentucky startup funds awarded to JKF. This manuscript has been released as a pre-print at BioRxiv, (Piedade et al., 2020).

\section{ACKNOWLEDGMENTS}

We thank Dr. Jessica Blackburn for providing space and reagents for cell culture work. We also thank Dr. Oliver Vocking for assistance with FWISH protocols. We thank Lucas Vieira for excellent zebrafish care and husbandry.

\section{SUPPLEMENTARY MATERIAL}

The Supplementary Material for this article can be found online at: https://www.frontiersin.org/articles/10.3389/fcell.2020. 594290/full\#supplementary-material

Supplementary Figure 1 | Siah1 overexpression decreases the number of rods and cones. Confocal stacks of heat shocked (HS) Tg[XOPS:GFP] (wildtype), Tg[hsp70:siah1]/Tg[XOPS:GFP] (siah1), and Tg[hsp70:siah1 $\Delta$ RING]/ Tg[XOPS:GFP], (siah1 $\triangle$ RING) embryos were collected analyzed in 3D for GFP fluorescence (A-C). Region analyzed and presented is outlined in yellow. Overexpression of Siah1 resulted in significantly fewer GFP + rod cells (G) Confocal stacks of heat shocked (HS) Tg[TaC:eGFP] (wildtype), Tg[hsp70:siah1]/

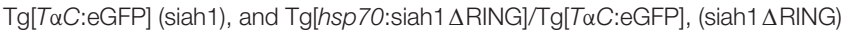
embryos were analyzed in 3D for GFP fluorescence (D-F). Region analyzed and presented is outlined in yellow. Overexpression of Siah1 resulted in significantly fewer GFP + cone cells $\mathbf{( H )}$. Scale bar $=50 \mu \mathrm{m}$.

Supplementary Figure 2 | Inner retinal neurons are not affected by Siah1 overexpression. Retinal cryosections from wildtype, Tg[hsp70:siah1] (siah1), and $\mathrm{Tg}[h s p 70: \operatorname{siah} 1 \Delta \mathrm{RING}]$ (siah1 $\Delta \mathrm{RING}$ ) embryos heat shocked $(\mathrm{HS}$ ) and analyzed for effects on retinal inner neurons using $\mathrm{HC}$. Retinal ganglion and amacrine cells were visualized and quantified using Huc/D staining (A-D). Bipolar cells were visualized and quantified using PKC $\alpha$ (E-H). Horizontal cells were observed and quantified using prox1 staining (I-L). DNA was stained with DAPI (blue). Scale bar = $50 \mu \mathrm{m}$. L, lens; ONL, outer nuclear layer; INL, Inner nuclear layer; GCL, ganglion cell layer; ON, optic nerve; D, Dorsal; and V, Ventral.

Supplementary Figure 3 | Siah1 does not affect retinal cell proliferation or cell cycle progression. Retinal cryosections from wildtype, Tg[hsp70:siah1] (siah1), and $\mathrm{Tg}[h s p 70$ :siah1 $\Delta \mathrm{RING}$ ( $\operatorname{siah1} \Delta \mathrm{RING}$ ) embryos heat shocked (HS) and analyzed for cell cycle status using PCNA (A-C) and PH3 (D-F) $\Vdash H C$ staining. Number of PCNA or $\mathrm{PH} 3$ positive cells did not significantly change upon Siah1 overexpression (G,H). DNA was stained with DAPI (blue). Scale bar $=50 \mu \mathrm{m}$. L, lens; ONL, outer nuclear layer; INL, Inner nuclear layer; GCL, ganglion cell layer; $\mathrm{ON}$, optic nerve; D, Dorsal; and V, Ventral.

Supplementary Figure 4 | Proteasome inhibition rescues Siah1 overexpression phenotype. Confocal stacks of heat shocked (HS) Tg[XOPS:GFP] (wildtype), Tg[hsp70:siah1]/Tg[XOPS:GFP] (siah1), and Tg[hsp70:siah1 $\triangle$ RING]/Tg[XOPS: 
GFP], (siah1 $\triangle \mathrm{RING}$ ) embryos treated with DMSO or MG132 were collected analyzed in 3D for GFP fluorescence. Quantification was restricted to the ventral retina (A-D). Treatment with MG132 prevented a significant decrease in GFP + rod cells compared to DMSO in siah1 HS embryos (E). Confocal stacks of heat shocked (HS) $\operatorname{Tg}[T \alpha C$ :eGFP] (wildtype), $\operatorname{Tg}[h s p 70$ :siah1] $/ \operatorname{Tg}[T \alpha C$ :eGFP] (siah1), and Tg[hsp70:siah1 $\Delta R I N G] / T g[T \alpha C: e G F P]$, (siah1 $\Delta R I N G)$ embryos treated with DMSO or MG132 were analyzed in 3D for GFP fluorescence. Quantification was restricted to the ventral retina (F-I). Treatment with MG132 prevented a significant decrease in GFP + cone cells compared to DMSO in siah1 HS embryos (J). Scale bar $=50 \mu \mathrm{m}$.

Supplementary Figure $\mathbf{5}$ | Time course of retinal apoptosis following siah1 or siah1 $\triangle$ RING heat shock. Retinal cryosections from heat shocked Tg[hsp70: siah1] (siah1), and Tg[hsp70:siah1 $\Delta \mathrm{RING}$ ( $(\operatorname{siah} 1 \Delta \mathrm{RING}$ ) embryos were analyzed

\section{REFERENCES}

Ackland, P., Resnikoff, S., and Bourne, R. (2017). World blindness and visual impairment: despite many successes, the problem is growing. Community Eye Heal. 30, 71-73.

Arno, G., Hull, S., Carss, K., Dev-Borman, A., Chakarova, C., Bujakowska, K., et al. (2016). Reevaluation of the retinal dystrophy due to recessive alleles of RGR with the discovery of a cis-acting mutation in CDHR1. Investig. Ophthalmol. Vis. Sci. 57, 4806-4813. doi: 10.1167/iovs.16-19687

Avanesov, A., and Malicki, J. (2010). Analysis of the retina in the zebrafish model. Methods Cell Biol. 100, 153-204. doi: 10.1016/B978-0-12-384892-5.00006-2

Ba-Abbad, R., Sergouniotis, P. I., Plagnol, V., Robson, A. G., Michaelides, M., Holder, G. E., et al. (2013). Clinical characteristics of early retinal disease due to CDHR1 mutation. Mol. Vis. 19, 2250-2259.

Babb, S. G., Kotradi, S. M., Shah, B., Chiappini-Williamson, C., Bell, L. N., Schmeiser, G., et al. (2005). Zebrafish R-cadherin (Cdh4) controls visual system development and differentiation. Dev. Dyn. 233, 930-945. doi: 10.1002/dvdy. 20431

Baker, S. A., and Kerov, V. (2013). Photoreceptor inner and outer segments. Curr. Top. Membr. 72, 231-265. doi: 10.1016/B978-0-12-417027-8.00007-6

Benati, D., Patrizi, C., and Recchia, A. (2019). Gene editing prospects for treating inherited retinal diseases. J. Med. Genet. 57, 437-444. doi: 10.1136/jmedgenet2019-106473

Bessenyei, B., and Oláh, É (2014). Clinical and genetic characteristics of craniosynostosis. Orv. Hetil. 155, 341-347. doi: 10.1556/oh.2014.29821

Biehlmaier, O., Neuhauss, S. C. F., and Kohler, K. (2003). Double cone dystrophy and RPE degeneration in the retina of the zebrafish gnn mutant. Investig. Ophthalmol. Vis. Sci. 44, 1287-1298. doi: 10.1167/iovs.02-0363

Bolz, H., Ebermann, I., and Gal, A. (2005). Protocadherin-21 (PCDH21), a candidate gene for human retinal dystrophies. Mol. Vis. 11, 929-933.

Brockerhoff, S. E., Hurley, J. B., Janssen-Bienhold, U., Neuhauss, S. C. F., Driever, W., and Dowling, J. E. (1995). A behavioral screen for isolating zebrafish mutants with visual system defects. Proc. Natl. Acad. Sci. U.S.A. 92, 1054510549. doi: $10.1073 /$ pnas. 92.23 .10545

Buch, P. K., Mihelec, M., Cottrill, P., Wilkie, S. E., Pearson, R. A., Duran, Y., et al. (2011). Dominant cone-rod dystrophy: a mouse model generated by gene targeting of the GCAP1/Gucala gene. PLoS One 6:e18089. doi: 10.1371/journal. pone. 0018089

Burgoyne, T., Meschede, I. P., Burden, J. J., Bailly, M., Seabra, M. C., Futter, C. E., et al. (2015). Rod disc renewal occurs by evagination of the ciliary plasma membrane that makes cadherin-based contacts with the inner segment. Proc. Natl. Acad. Sci. U.S.A. 112, 15922-15927. doi: 10.1073/pnas.1509285113

Centanin, L., and Wittbrodt, J. (2014). Retinal neurogenesis. Development 141, 241-244. doi: $10.1242 / \mathrm{dev} .083642$

Clendenon, S. G., Sarmah, S., Shah, B., Liu, Q., and Marrs, J. A. (2012). Zebrafish cadherin-11 participates in retinal differentiation and retinotectal axon projection during visual system development. Dev. Dyn. 241, 442-454. doi: $10.1002 /$ dvdy.23729

Cohen, B., Chervinsky, E., Jabaly-Habib, H., Shalev, S. A., Briscoe, D., and Ben-Yosef, T. (2012). A novel splice site mutation of CDHR1 in a consanguineous israeli christian arab family segregating autosomal recessive cone-rod dystrophy. Mol. Vis. 18, 2915-2921. using $\mathrm{HC}$ for apoptosis using TUNEL staining (red) at various time points (A-E'). DNA was visualized using DAPI (blue). Heat shock induction of siah1 $\triangle R I N G$ expression had little effect on induction of retinal apoptosis between 52-72 hpf (A-F). Heat shock induction of siah1 resulted in a significant increase of apoptotic cells in the retina beginning at $60 \mathrm{hpf}$ and up to $72 \mathrm{hpf}$ compared to siah1 $\triangle$ RING (A'-E', F). Each point on the graph represents counts from individual embryos (F).

Supplementary Figure 6 | Genomic PCR screen for CRISPR induced insertion or deletion. Genomic DNA collected from injected embryos was used to amplify $\sim 250$ bp amplicon from cdhr1a coding sequence. Golden crRNA control injected embryos all display a strong 250 bp band (embryos 1-3, 14-16). When examining PCR products from cdhr1a crRNA injected embryos various combinations of insertion or deletion products were amplified (embryos 4-13 and 17-26.

Coomer, C. E., and Morris, A. C. (2018). Capn5 expression in the healthy and regenerating zebrafish retina. Investig. Opthalmol. Vis. Sci. 59, 3643. doi: 10. 1167/iovs.18-24278

Duncan, J. L., Roorda, A., Navani, M., Vishweswaraiah, S., Syed, R., Soudry, S., et al. (2012). Identification of a novel mutation in the CDHR1 gene in a family with recessive retinal degeneration. Arch. Ophthalmol. 130, 1301-1308. doi: 10.1001/archophthalmol.2012.1906

Eblimit, A., Agrawal, S. A., Thomas, K., Anastassov, I. A., Abulikemu, T., Moayedi, Y., et al. (2018). Conditional loss of Spata7 in photoreceptors causes progressive retinal degeneration in mice. Exp. Eye Res. 166, 120-130. doi: 10.1016/j.exer. 2017.10.015

Fadool, J., and Dowling, J. (2008). Zebrafish: a model system for the study of eye genetics. Prog. Retin. Eye Res. 27, 89-110. doi: 10.1016/j.preteyeres.2007.08.002

Fadool, J. M. (2003). Development of a rod photoreceptor mosaic revealed in transgenic zebrafish. Dev. Biol. 258, 277-290. doi: 10.1016/s0012-1606(03) 00125-8

Fu, J., Ma, L., Cheng, J., Yang, L., Wei, C., Fu, S., et al. (2018). A novel, homozygous nonsense variant of the CDHR1 gene in a Chinese family causes autosomal recessive retinal dystrophy by NGS-based genetic diagnosis. J. Cell. Mol. Med. 22, 5662-5669. doi: 10.1111/jcmm.13841

Gill, J. S., Georgiou, M., Kalitzeos, A., Moore, A. T., and Michaelides, M. (2019). Progressive cone and cone-rod dystrophies: clinical features, molecular genetics and prospects for therapy. Br. J. Ophthalmol. 103, 711-720. doi: 10.1136/ bjophthalmol-2018-313278

Goldberg, A. F. X., Moritz, O. L., and Williams, D. S. (2016). Molecular basis for photoreceptor outer segment architecture. Prog. Retin. Eye Res. 55, 52-81. doi: $10.1016 /$ j.preteyeres.2016.05.003

Hamel, C. P. (2007). Cone rod dystrophies. Orphanet J. Rare Dis. 2, 1-7.

Henderson, R. H., Li, Z., Abd El Aziz, M. M., Mackay, D. S., Eljinini, M. A., Zeidan, M., et al. (2010). Biallelic mutation of protocadherin-21 (PCDH21) causes retinal degeneration in humans. Mol. Vis. 16, 46-52.

Hohman, T. C. (2016). Hereditary retinal dystrophy. Handb. Exp. Pharmacol. 242, 337-367. doi: 10.1007/164_2016_91

Hoon, M., Okawa, H., Della Santina, L., and Wong, R. O. L. (2014). Functional architecture of the retina: development and disease. Prog. Retin. Eye Res. 42, 44-84. doi: $10.1016 /$ j.preteyeres.2014.06.003

Hoshijima, K., Jurynec, M. J., Shaw, D. K., Jacobi, A. M., Behlke, M. A., and Grunwald, D. J. (2019). Highly efficient CRISPR-cas9-based methods for generating deletion mutations and F0 embryos that lack gene function in zebrafish. Dev. Cell. 51, 645-657. doi: 10.1016/j.devcel.2019.10.004

Kaewkhaw, R., Kaya, K. D., Brooks, M., Homma, K., Zou, J., Chaitankar, V., et al. (2015). Transcriptome dynamics of developing photoreceptors in threedimensional retina cultures recapitulates temporal sequence of human cone and rod differentiation revealing cell surface markers and gene networks. Stem Cells 33, 3504-3518. doi: 10.1002/stem.2122

Kawamura, S., and Tachibanaki, S. (2008). Rod and cone photoreceptors: molecular basis of the difference in their physiology. Comp. Biochem. Physiol. Part A Mol. Integr. Physiol. 150, 369-377. doi: 10.1016/j.cbpa.2008.04.600

Kennedy, B. N., Alvarez, Y., Brockerhoff, S. E., Stearns, G. W., Sapetto-Rebow, B., Taylor, M. R., et al. (2007). Identification of a zebrafish cone photoreceptorspecific promoter and genetic rescue of achromatopsia in the nof mutant. Investig. Opthalmology Vis. Sci. 48, 522. doi: 10.1167/iovs.06-0975 
Kocaoglu, O. P., Liu, Z., Zhang, F., Kurokawa, K., Jonnal, R., and Miller, D. T. (2016). Photoreceptor disc shedding in the living human eye. Biomed. Opt. Express 7:4554. doi: 10.1364/boe.7.004554

Larison, K. D., and Bremiller, R. (1990). Early onset of phenotype and cell patterning in the embryonic zebrafish retina. Development 109, 567-576.

Lauter, G., Söll, I., and Hauptmann, G. (2011). Two-color fluorescent in situ hybridization in the embryonic zebrafish brain using differential detection systems. BMC Dev. Biol. 11:43. doi: 10.1186/1471-213x-11-43

Lele, Z. A., Folchert, M Concha, Rauch, G. J., Geisler, R., Rosa, F., Wilson, S. W., et al. (2002). parachute/n-cadherin is required for morphogenesis and maintained integrity of the zebrafish neural tube. Development 129, 3281-3294.

Lu, Z., Hu, X., Liu, F., Soares, D. C., Liu, X., Yu, S., et al. (2017). Ablation of EYS in zebrafish causes mislocalisation of outer segment proteins, F-actin disruption and cone-rod dystrophy. Sci. Rep. 7, 1-12.

Malicki, J., Jo, H., and Pujic, Z. (2003). Zebrafish N-cadherin, encoded by the glass onion locus, plays an essential role in retinal patterning. Dev. Biol. 259, 95-108. doi: 10.1016/s0012-1606(03)00181-7

Masai, I., Stemple, D. L., Okamoto, H., and Wilson, S. W. (2000). Midline signals regulate retinal neurogenesis in zebrafish. Neuron 27, 251-263. doi: 10.1016/ s0896-6273(00)00034-9

Morris, A., and Fadool, J. (2005). Studying rod photoreceptor development in zebrafish. Physiol. Behav. 86, 306-313. doi: 10.1016/j.physbeh.2005.08.020

Morris, A. C., Scholz, T., and Fadool, J. M. (2008). Rod progenitor cells in the mature zebrafish retina. Adv. Exp. Med. Biol. 613, 361-368. doi: 10.1007/9780-387-74904-4_42

Morris, A. C., Schroeter, E. H., Bilotta, J., Wong, R. O. L., and Fadool, J. M. (2005). Cone survival despite rod degeneration in XOPS-mCFP transgenic zebrafish. Investig. Opthalmology Vis. Sci. 46, 4762. doi: 10.1167/iovs.05-0797

Nikopoulos, K., Avila-Fernandez, A., Corton, M., Lopez-Molina, M. I, Perez-Carro, R., Bontadelli, L., et al. (2015). Identification of two novel mutations in CDHR1 in consanguineous Spanish families with autosomal recessive retinal dystrophy. Sci. Rep. 5:13902.

Ostergaard, E., Batbayli, M., Duno, M., Vilhelmsen, K., and Rosenberg, T. (2010). Mutations in PCDH21 cause autosomal recessive cone-rod dystrophy. J. Med. Genet. 47, 665-669. doi: 10.1136/jmg.2009.069120

Parry, D. A., Toomes, C., Bida, L., Danciger, M., Towns, K. V., McKibbin, M., et al. (2009). Loss of the metalloprotease ADAM9 leads to cone-rod dystrophy in humans and retinal degeneration in mice. Am. J. Hum. Genet. 84, 683-691. doi: 10.1016/j.ajhg.2009.04.005

Piedade, W. P., Titialii-Torres, K., Morris, A. C., and Famulski, J. K. (2020). Proteasome-mediated regulation of Cdhrla by Siah1 modulates photoreceptor development and survival in zebrafish. bioRxiv 098350. doi: 10.1101/2020.05. 15.098350

Piedade, W. P., Veith, S., and Famulski, J. K. (2019). Ubiquitin-mediated proteasome degradation regulates optic fissure fusion. Biol. Open 8:bio044974. doi: 10.1242/bio.044974

Pontoriero, G. F., Smith, A. N., Miller, L.-A. D., Radice, G. L., West-Mays, J. A., and Lang, R. A. (2009). Co-operative roles for E-cadherin and N-cadherin during lens vesicle separation and lens epithelial cell survival. Dev. Biol. 326, 403-417. doi: 10.1016/j.ydbio.2008.10.011

Pujic, Z., and Malicki, J. (2001). Mutation of the zebrafish glass onion locus causes early cell-nonautonomous loss of neuroepithelial integrity followed by severe neuronal patterning defects in the retina. Dev. Biol. 234, 454-469. doi: 10.1006/ dbio.2001.0251

Purves, D., Augustine, G. J., Fitzpatrick, D., Katz, L. C., LaMantia, A.-S., McNamara, J. O., et al. (2001). "Thalamocortical Interactions," in Neuroscience, eds D. Purves, et al. (Sunderland, MA: Sinauer Associates.

Qu, Z., Yimer, T. A., Xie, S., Wong, F., Yu, S., Liu, X., et al. (2019). Knocking out lca5 in zebrafish causes cone-rod dystrophy due to impaired outer segment protein trafficking. Biochim. Biophys. Acta Mol. Basis Dis. 1865, 2694-2705. doi: 10.1016/j.bbadis.2019.07.009

Rattner, A., Chen, J., and Nathans, J. (2004). Proteolytic shedding of the extracellular domain of photoreceptor cadherin. Implications for outer segment assembly. J. Biol. Chem. 279, 42202-42210. doi: 10.1074/jbc.m4079 28200

Rattner, A., Smallwood, P. M., Williams, J., Cooke, C., Savchenko, A., Lyubarsky, A., et al. (2001). A photoreceptor-specific cadherin is essential for the structural integrity of the outer segment and for photoreceptor survival. Neuron 32, 775-786. doi: 10.1016/s0896-6273(01)00531-1

Raymond, P. A., Barthel, L. K., and Curran, G. A. (1995). Developmental patterning of rod and cone photoreceptors in embryonic zebrafish. J. Comp. Neurol. 359, 537-550. doi: 10.1002/cne.903590403

Riera, M., Navarro, R., Ruiz-nogales, S., Mendez, P., Bures-jelstrup, A., Corcóstegui, B., et al. (2017). Whole exome sequencing using Ion Proton system enables reliable genetic diagnosis of inherited retinal dystrophies. Sci. Rep. 7, $1-13$.

Schmitt, E. A., and Dowling, J. E. (1996). Comparison of topographical patterns of ganglion and photoreceptor cell differentiation in the retina of the zebrafish,Danio rerio. J. Comp. Neurol. 371, 222-234. doi: 10.1002/(sici)10969861(19960722)371:2<222::aid-cne3>3.0.co;2-4

Stingl, K., Mayer, A. K., Llavona, P., Mulahasanovic, L., Rudolph, G., Jacobson, S. G., et al. (2017). CDHR1 mutations in retinal dystrophies. Sci. Rep. 7:6992.

Sullivan, L., Bowne, S., and Daiger, S. (2020). RetNet-Retinal Information Network. Available online at: https://sph.uth.edu/retnet/ (accessed May 11, 2020).

van Driel, D., Provis, J. M., and Billson, F. A. (1990). Early differentiation of ganglion, amacrine, bipolar, and muller cells in the developing fovea of human retina. J. Comp. Neurol. 291, 203-219. doi: 10.1002/cne.902910205

Wan, Y., Almeida, A. D., Rulands, S., Chalour, N., Muresan, L., Wu, Y., et al. (2016). The ciliary marginal zone of the zebrafish retina: clonal and timelapse analysis of a continuously growing tissue. Development 143, 1099-1107. doi: $10.1242 /$ dev.133314

Conflict of Interest: The authors declare that the research was conducted in the absence of any commercial or financial relationships that could be construed as a potential conflict of interest.

Copyright (C) 2020 Piedade, Titialii-Torres, Morris and Famulski. This is an openaccess article distributed under the terms of the Creative Commons Attribution License (CC BY). The use, distribution or reproduction in other forums is permitted, provided the original author(s) and the copyright owner(s) are credited and that the original publication in this journal is cited, in accordance with accepted academic practice. No use, distribution or reproduction is permitted which does not comply with these terms. 